\title{
Rigidification of higher categorical structures
}

\author{
Giovanni Caviglia \\ GEOFFROY HOREL
}

\begin{abstract}
Given a limit sketch in which the cones have a finite connected base, we show that a model structure of "up to homotopy" models for this limit sketch in a suitable model category can be transferred to a Quillen-equivalent model structure on the category of strict models. As a corollary of our general result, we obtain a rigidification theorem which asserts in particular that any $\Theta_{n}$-space in the sense of Rezk is levelwise equivalent to one that satisfies the Segal conditions on the nose. There are similar results for dendroidal spaces and $n$-fold Segal spaces.
\end{abstract}

18C30, 18D35, 55U35; 18D05, 18D50

\section{Introduction}

This paper is concerned with the problem of rigidification for higher categorical structures. Usually, the correct definition of a higher categorical object is the one where the relations are required to hold as weakly as possible. However, these objects can sometimes be partially rigidified to equivalent objects for which some of the relations hold strictly. The simplest example of this phenomenon can be seen on monoidal categories. The standard definition of a monoidal category involves associators and unitors that insure that any two ways of tensoring a finite sequence of objects are uniquely isomorphic. On the other hand, it is known that any monoidal category is equivalent to a monoidal category in which the tensor product is unital and associative on the nose, meaning that all the possible ways of tensoring a finite sequence of objects are equal. More generally, any bicategory is equivalent to a 2-category. However, this is a lucky accident that does not happen for higher-dimensional higher categories. For instance, a trigroupoid encoding the 3-type of the 2-sphere cannot be rigidified to a strict 3-groupoid; see Simpson [23, Section 2.7]. These problems become more and more intractable as the dimension gets bigger.

One consequence of our main result can be vaguely phrased by saying that the problem of rigidification of higher categories only comes from the invertible cells, and as long as one does not to try to rigidify them, it should be possible to get a strict model of the higher category. A more precise statement of what we prove is that the homotopy 
theory of $(\infty, n)$-categories is equivalent to that of strict $n$-categories internal to Kan complexes. According to the homotopy hypothesis, the homotopy theory of any coherent-enough notion of $\infty$-groupoid should be equivalent to the homotopy theory of $\mathrm{CW}$-complexes up to homotopy equivalences. It is well-known that the homotopy theory of Kan complexes is equivalent to that of $\mathrm{CW}$-complexes, and many mathematicians actually take Kan complexes as a definition of $\infty$-groupoids.

Our method for proving this rigidification result is to put a model structure on the category of $n$-categories internal to simplicial sets that is equipped with a Quillen equivalence to the model category of $\Theta_{n}$-spaces with the Rezk model structure. It is widely accepted by the mathematical community that $\Theta_{n}$-spaces form a good model of $(\infty, n)$ categories. In [7], Bergner and Rezk compare this model to other reasonable models of $(\infty, n)$-categories. This model also satisfies the axiomatization of $(\infty, n)$-categories given by Barwick and Schommer-Pries in [3].

A partial result in this direction was obtained by the second author in [14]. One of the main results of that paper was to prove an equivalence between the model structure of complete Segal spaces and a model structure on categories internal to simplicial sets. In that case, the rigidification result is, of course, not very surprising because by work of Bergner [6] we even know that a complete Segal space can be rigidified to a simplicially enriched category.

When we tried to generalize the result of [14] to $(\infty, n)$-categories, we realized not only that it could be done, but also that the proof used very little about the category of $n$-categories. The main point is that $n$-categories form a locally presentable category, and that there is a full subcategory $\Theta_{n}$ such that the associated nerve construction $n$ Cat $\rightarrow$ Set $^{\Theta_{n}^{\text {op }}}$ is fully faithful and preserves coproducts and filtered colimits.

We can thus abstract this situation and consider pairs consisting of a locally presentable category $\mathbf{X}$ with a full subcategory $\Xi$ satisfying the following assumptions:

Assumptions 1.1 $\quad \Xi$ is dense in $\mathbf{X}$; ie the functor $N: \mathbf{X} \rightarrow \operatorname{Set}^{\mathbf{\Xi}^{\text {op }}}$ sending $X$ to $\xi \mapsto \mathbf{X}(\xi, X)$ is fully faithful.

- For any $\xi$ in $\Xi$, the functor $\mathbf{X}(\xi,-)$ preserves filtered colimits.

- For any $\xi$ in $\Xi$, the functor $\mathbf{X}(\xi,-)$ preserves finite coproducts.

It turns out that the category of $n$-categories is not the only interesting example of such a situation. For instance, one can take $\mathbf{X}$ to be the category of colored operads and $\Xi$ to be its subcategory $\Omega$. One can also take $\mathbf{X}$ to be the category $\mathbf{C a t}^{\otimes n}$ of $n$-fold categories and the subcategory $\Delta^{n}$. In fact, we prove in Proposition 4.4 that given a locally presentable category $\mathbf{X}$, the existence of a full subcategory $\Xi$ satisfying 
Assumptions 1.1 are equivalent to the fact that $\mathbf{X}$ is the category of models for a finite connected limit sketch.

Our main result is given by the following theorem.

Theorem 5.9 Let $(\mathbf{X}, \Xi)$ be a pair satisfying Assumptions 1.1, and $\mathbf{S}$ a Grothendieck topos equipped with a combinatorial model structure in which the cofibrations are monomorphisms. The projective model structure on $\mathbf{S}^{\mathbf{\Xi}^{\text {op }}}$, as well as any of its left Bousfield localizations, can be lifted to $\mathbf{S} \otimes \mathbf{X}$, the category of objects of $\mathbf{X}$ internal to $\mathbf{S}$, via the nerve functor. The resulting adjunction between $\mathbf{S}^{\Xi^{\text {op }}}$ and $\mathbf{S} \otimes \mathbf{X}$ is moreover a Quillen equivalence.

We advise the reader to not read this paper linearly, but rather, start from the last section where the most interesting applications are developed, and refer to the previous sections as needed. In particular, Theorems 7.5, 7.6 and 7.7 examine the consequences of the above theorem to the theories of operads, $n$-categories and $n$-fold categories, respectively. The main theorem implies rigidification results which can be expressed by saying that any homotopy operad, $n$-category or $n$-fold category internal to $\mathbf{S}$ is levelwise equivalent to one which is strict.

Acknowledgements Caviglia wishes to thank Dimitri Ara for the suggestion to look at this problem, Ieke Moerdijk for his support and Javier J Gutiérrez, Simon Henry and Joost Nuiten for many insightful discussions.

Horel wishes to thank the Hausdorff Institute for Mathematics in Bonn for providing an excellent work environment in the summer of 2015 during which most of this paper was written, and also Claudia Scheimbauer and Chris Schommer-Pries for their interest in this project.

We both thank the anonymous referee for several helpful comments.

\section{A few facts on locally presentable categories}

Let $\lambda$ be a regular cardinal. Given a small category $C$ with all $\lambda$-small colimits, we denote by $\operatorname{lnd}_{\lambda}(C)$ the category of functors $C^{\text {op }} \rightarrow$ Set that preserve $\lambda$-small limits. When $\lambda=\omega$ is the smallest infinite cardinal, we write Ind instead of $\operatorname{lnd}_{\omega}$.

Definition 2.1 A $\lambda$-locally presentable category is a category $\mathbf{C}$ that is equivalent to $\operatorname{Ind}_{\lambda}(A)$, where $A$ is a small category with all $\lambda$-small colimits. A locally presentable category is a category that is $\lambda$-locally presentable for some $\lambda$. 
Proposition 2.2 Let $\mathbf{C}$ be a category and $I: \mathbf{C} \rightarrow \operatorname{Fun}(A$, Set $)$ be a fully faithful right adjoint that preserves $\lambda$-filtered colimits. Then $\mathbf{C}$ is $\lambda$-locally presentable.

Proof See [1, Theorem 1.46].

If $\mathbf{C}$ and $\mathbf{D}$ are two locally presentable categories, we denote by $\mathbf{C} \otimes \mathbf{D}$ their tensor product in the category of locally presentable categories. This is a locally presentable category with a functor $\mathbf{C} \times \mathbf{D} \rightarrow \mathbf{C} \otimes \mathbf{D}$ that preserves colimits in each variable separately and which is initial with this property.

We now recall how this tensor product is constructed explicitly. It is not restrictive to suppose that $\mathbf{C}$ and $\mathbf{D}$ are $\lambda$-presentable categories for some regular cardinal $\lambda$. Then $\mathbf{C} \simeq \operatorname{Ind}_{\lambda}(A)$ and $\mathbf{D} \simeq \operatorname{lnd}_{\lambda}(B)$ for some small full subcategory $A$ (resp. $B$ ) of $\mathbf{C}$ (resp. D). We can then construct the category $\mathbf{C} \otimes \mathbf{D}:=\operatorname{Fun}^{\lambda R, \lambda R}\left(A^{\mathrm{op}} \times B^{\mathrm{op}}\right.$, Set $)$. This is the full subcategory of Fun $\left(A^{\mathrm{op}} \times B^{\mathrm{op}}\right.$, Set $)$ spanned by the functors that preserve $\lambda$-small limits in each variable. This category is also equivalent to the category Fun $^{R, R}\left(\mathbf{C}^{\mathrm{op}} \times \mathbf{D}^{\mathrm{op}}\right.$, Set $)$ of functors $\mathbf{C}^{\mathrm{op}} \times \mathbf{D}^{\mathrm{op}} \rightarrow$ Set preserving limits separately in each variable. Note however that this second definition does not make it obvious that $\mathbf{C} \otimes \mathbf{D}$ has small hom-sets.

The functor $\mathbf{C} \times \mathbf{D} \rightarrow \mathbf{C} \otimes \mathbf{D}$ sends $(c, d)$ to the functor $c \otimes d \in \operatorname{Fun}\left(\mathbf{C}^{\mathrm{op}} \times \mathbf{D}^{\mathrm{op}}\right.$, Set $)$ such that $c \otimes d\left(c^{\prime}, d^{\prime}\right)=\mathbf{C}\left(c^{\prime}, c\right) \times \mathbf{D}\left(d^{\prime}, d\right)$ for every $\left(c^{\prime}, d^{\prime}\right) \in \mathbf{C}^{\mathrm{op}} \times \mathbf{D}^{\mathrm{op}}$. It is easy to verify that $c \otimes d$ preserves limits in each variable, and hence we have indeed constructed a functor $\mathbf{C} \times \mathbf{D} \rightarrow \mathbf{C} \otimes \mathbf{D}$.

Using the fact that the map $\mathbf{D} \rightarrow$ Fun $^{R}\left(\mathbf{D}^{\text {op }}\right.$, Set) sending $d$ to the limit preserving functor $\mathbf{D}(-, d)$ from $\mathbf{D}^{\text {op }}$ to Set is an equivalence of categories, we see that $\mathbf{C} \otimes \mathbf{D}$ is also equivalent to the category $\operatorname{Fun}^{R}\left(\mathbf{C}^{\text {op }}, \mathbf{D}\right)$. Note that with this last description, the commutativity of the tensor product is not obvious. Through the equivalence $\mathbf{C} \otimes \mathbf{D} \simeq \operatorname{Fun}^{R}\left(\mathbf{C}^{\mathrm{op}}, \mathbf{D}\right)$, the object $c \otimes d$ is the limit preserving functor $\mathbf{C}^{\mathrm{op}} \rightarrow \mathbf{D}$ such that we have a natural isomorphism

$$
\operatorname{Fun}^{R}\left(\mathbf{C}^{\mathrm{op}}, \mathbf{D}\right)(c \otimes d, F) \cong \mathbf{D}(d, F(c))
$$

We now study the functoriality of this tensor product. Let us assume that we are given an adjunction $u^{*}: \mathbf{C} \rightleftarrows \mathbf{D}: u_{*}$ between locally presentable categories, and let $\mathbf{Z}$ be any locally presentable category. We can construct a functor $u_{*} \otimes$ id: $\mathbf{D} \otimes \mathbf{Z} \rightarrow \mathbf{C} \otimes \mathbf{Z}$ by identifying $\mathbf{C} \otimes \mathbf{Z}$ with Fun ${ }^{R}\left(\mathbf{C}^{\mathrm{op}}, \mathbf{Z}\right)$ and $\mathbf{D} \otimes \mathbf{Z}$ with Fun $^{R}\left(\mathbf{D}^{\mathrm{op}}, \mathbf{Z}\right)$, and taking the functor induced by precomposition with $\left(u^{*}\right)^{\text {op }}$. We can also construct the functor $\mathrm{id} \otimes u_{*}: \mathbf{Z} \otimes \mathbf{D} \rightarrow \mathbf{Z} \otimes \mathbf{C}$ obtained by identifying $\mathbf{Z} \otimes \mathbf{C}$ with $\mathrm{Fun}^{R}\left(\mathbf{Z}^{\mathrm{op}}, \mathbf{C}\right)$ and $\mathbf{Z} \otimes \mathbf{D}$ with $\operatorname{Fun}^{R}\left(\mathbf{Z}^{\mathrm{op}}, \mathbf{D}\right)$, and taking the functor induced by postcomposition with $u_{*}$. 
Lemma 2.3 Let $u^{*}: \mathbf{C} \rightleftarrows \mathbf{D}: u_{*}$ be an adjunction between locally presentable categories, and let $\mathbf{Z}$ be another locally presentable category. The diagram

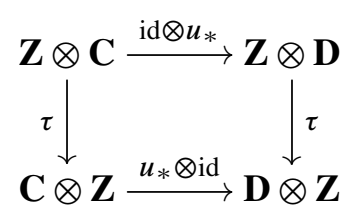

commutes up to a natural isomorphism.

Proof Let $F \in \operatorname{Fun}^{R}\left(\mathbf{Z}^{\text {op }}, \mathbf{C}\right) \simeq \mathbf{Z} \otimes \mathbf{C}$. Using formula (2-1), we see that the functor $\tau\left(\mathrm{id} \otimes u_{*}\right)(F)$ sends $(d, z) \in \mathbf{D}^{\mathrm{op}} \times \mathbf{Z}^{\text {op }}$ to $\mathbf{D}\left(d, u_{*} F(z)\right)$. On the other hand, the functor $\left(u_{*} \otimes \mathrm{id}\right) \tau(F)$ sends $(d, z)$ to $\mathbf{D}\left(u^{*}(d), F(z)\right)$. Thus the two functors $\left(u_{*} \otimes \mathrm{id}\right) \tau(F)$ and $\tau\left(\mathrm{id} \otimes u_{*}\right)(F)$ are isomorphic, and moreover, this isomorphism can be chosen to be functorial in $F$. This proves the commutativity of the square.

Keeping the same notation as before, we can also construct a functor $\mathbf{Z} \times \mathbf{C} \rightarrow \mathbf{Z} \otimes \mathbf{D}$ sending $(z, c)$ to $z \otimes u^{*}(c)$. This functor preserves colimits in both variables and hence, by the universal property of the tensor product, induces a colimit preserving functor $\mathbf{Z} \otimes \mathbf{C} \rightarrow \mathbf{Z} \otimes \mathbf{D}$ that we denote by id $\otimes u^{*}$.

Lemma 2.4 The functor id $\otimes u_{*}$ is right adjoint to id $\otimes u^{*}$.

Proof Let $F: \mathbf{Z}^{\text {op }} \rightarrow \mathbf{D}$ be an object of $\mathbf{Z} \otimes \mathbf{D} \simeq \operatorname{Fun}^{R}\left(\mathbf{Z}^{\mathrm{op}}, \mathbf{D}\right)$. Using (2-1), we find a sequence of natural isomorphisms,

$$
\begin{aligned}
\mathbf{Z} \otimes \mathbf{D}\left(\left(\mathrm{id} \otimes u^{*}\right)(z \otimes c), F\right) & \cong \mathbf{Z} \otimes \mathbf{D}\left(z \otimes u^{*} c, F\right) \\
& \cong \mathbf{Z}\left(z, F\left(u^{*} c\right)\right) \cong \mathbf{Z} \otimes \mathbf{C}\left(z \otimes c,\left(\operatorname{id} \otimes u_{*}\right) F\right),
\end{aligned}
$$

which proves the desired result.

Corollary 2.5 Let $\mathbf{C}$ and $\mathbf{D}$ be locally presentable categories and let $u^{*}: \mathbf{C} \rightleftarrows \mathbf{D}: u_{*}$ be an adjunction between them. Suppose that $u^{*}$ commutes with $\lambda$-small limits. Let $\mathbf{Z} \simeq \operatorname{Ind}_{\lambda}(A)$ be a $\lambda$-locally presentable category. Then the functor $\mathrm{id} \otimes u^{*}$ is isomorphic to the composite

$$
\mathbf{Z} \otimes \mathbf{C} \simeq \operatorname{Fun}^{\lambda R}\left(A^{\mathrm{op}}, \mathbf{C}\right) \stackrel{u^{*} \circ-}{\longrightarrow} \operatorname{Fun}^{\lambda R}\left(A^{\mathrm{op}}, \mathbf{D}\right) \simeq \mathbf{Z} \otimes \mathbf{D},
$$

where the middle map is given by postcomposition with $u^{*}$ 
Proof By Lemma 2.4 and the uniqueness of a left adjoint, it suffices to prove that postcomposition with $u^{*}$ is left adjoint to id $\otimes u_{*}$. But since id $\otimes u_{*}$ is isomorphic to the composite

$$
\mathbf{Z} \otimes \mathbf{D} \simeq \operatorname{Fun}^{\lambda R}\left(A^{\mathrm{op}}, \mathbf{D}\right) \stackrel{u_{*} \mathrm{o}-}{\longrightarrow} \operatorname{Fun}^{\lambda R}\left(A^{\mathrm{op}}, \mathbf{C}\right) \simeq \mathbf{Z} \otimes \mathbf{C},
$$

the result is obvious.

\section{The setup}

Let $\mathbf{X}$ be a category with all colimits and let $\Xi$ be a small full subcategory of $\mathbf{X}$. We assume that the pair $(\mathbf{X}, \Xi)$ satisfies Assumptions 1.1. Denote by $S: \mathbf{S e t}^{\Xi \text { op }} \rightarrow \mathbf{X}$ the left Kan extension of the inclusion $\boldsymbol{\Xi} \rightarrow \mathbf{X}$ along the Yoneda embedding $y: \Xi \rightarrow \mathbf{S e t}^{\boldsymbol{\Xi}^{\text {op }}}$. The functor $S$ always has a right adjoint, the nerve functor, that we denote by $N$. Concretely, if $A$ is an object of $\mathbf{X}$, then $N A(\xi)=\mathbf{X}(\xi, A)$.

Using the first point of Assumptions 1.1, we see that $\mathbf{X}$ is a full reflective subcategory of Set $^{\Xi^{o p}}$ via the adjunction $(S, N)$. In particular, according to Proposition 2.2, $\mathbf{X}$ is an $\omega$-locally presentable category, and hence has all small limits. The limits in $\mathbf{X}$ are created by the nerve functor. Under the other two points of Assumptions 1.1, we find that the finite coproducts and filtered colimits in $\mathbf{X}$ are also created by the nerve functor.

Example 3.1 The main examples that we have in mind are the following; they will be developed in detail in the last section:

(1) The category $\mathbf{X}$ is the category Cat of small categories. The category $\Xi$ is the category $\Delta$ of finite linearly ordered sets. The functor $N$ is the usual nerve functor from Cat to simplicial sets.

(2) The category $\mathbf{X}$ is the category $\mathbf{O p}$ of small colored operads in sets. The category $\Xi$ is the category $\Omega$ of dendrices. The functor $N$ is the dendroidal nerve functor from colored operads to dendroidal sets.

(3) $\mathbf{X}$ is the category of $n$-fold categories, and $\Xi$ is $\Delta^{n}$.

(4) $\mathbf{X}$ is the category of $n$-categories, and $\Xi$ is Joyal's theta category $\Theta_{n}$.

(5) $\mathbf{X}$ is the category of models for a finite connected limit sketch. In that case, the full subcategory $\Xi$ can be constructed as explained in Section 4.

We also have a category of geometric objects $\mathbf{S}$ that we generically call "spaces". We make the following assumption on $\mathbf{S}$. 
Assumption 3.2 We assume that $\mathbf{S}$ is a Grothendieck topos equipped with a combinatorial model structure in which the cofibrations are monomorphisms.

Example 3.3 The prototypical example of a category $\mathbf{S}$ satisfying Assumption 3.2 is the category $\operatorname{Set}^{\Delta^{\text {op }}}$ of simplicial sets with its standard model structure. If $I$ is a small category, the category $\operatorname{Set}^{\Delta^{\mathrm{op}} \times I^{\mathrm{op}}}$ of simplicial presheaves on $I$, with its projective or injective model structure, also satisfies our hypothesis. If $I$ is a site, then the Joyal model structure (see [15, Theorem 5.9]) on the category of simplicial sheaves on $I$, denoted $\mathbf{S h}\left(I, \mathbf{S e t}^{\Delta^{\mathrm{op}}}\right)$, also satisfies the hypothesis.

It is also the case that if $\mathbf{S}$ is a category equipped with a model structure satisfying Assumption 3.2, the same is true for any Bousfield localization of $\mathbf{S}$. Thus, for any site $I$, the category $\left(\mathbf{S e t}^{\Delta^{\mathrm{op}}}\right)^{I}$ with the local model structure of Jardine [15, Theorem 5.8] also satisfies Assumption 3.2. Another example of interest that satisfies Assumption 3.2 is the model category of motivic spaces. It is obtained by localizing the Jardine model structure of presheaves over the Nisnevich site of a field $k$ at the $\mathbb{A}^{1}$-equivalences.

However, note that the usual model structure on topological spaces does not satisfy Assumption 3.2. The main issue is that topological spaces do not form a topos.

Proposition 3.4 Let $(\mathbf{X}, \Xi)$ be a pair satisfying Assumptions 1.1. Let $\pi: \mathbf{R} \rightarrow \mathbf{S}$ be a geometric morphism between Grothendieck toposes. The diagram

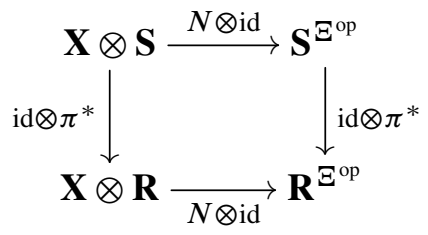

commutes up to isomorphism.

Proof We identify $\mathbf{X} \otimes \mathbf{S}$ with $\mathrm{Fun}^{R}\left(\mathbf{X}^{\mathrm{op}}, \mathbf{S}\right)$. By Lemma 2.3, both instances of $N \otimes$ id in the diagram are given by precomposition with $S$, and by Corollary 2.5, both instances of $\mathrm{id} \otimes \pi^{*}$ in the diagram are given by postcomposition with $\pi^{*}$.

Remark 3.5 Recall that a geometric morphism $\pi: \mathbf{R} \rightarrow \mathbf{S}$ between toposes is surjective if and only if the inverse image functor $\pi^{*}$ is conservative. In a topos, a map $X \rightarrow Y$ is a monomorphism if and only if the induced map $X \rightarrow X \times_{Y} X$ is an isomorphism. Given a surjective geometric morphism $\pi: \mathbf{R} \rightarrow \mathbf{S}$, the functor $\pi^{*}: \mathbf{S} \rightarrow \mathbf{R}$ preserves finite limits and is conservative; therefore, it preserves and reflects monomorphisms. 
Proposition 3.6 Let $(\mathbf{X}, \Xi)$ be a pair of categories satisfying Assumptions 1.1, and $\mathbf{S}$ a Grothendieck topos. The functor $\mathrm{id} \otimes N: \mathbf{S} \otimes \mathbf{X} \rightarrow \mathbf{S}^{\Xi^{\text {op }}}$ exhibits $\mathbf{S} \otimes \mathbf{X}$ as a reflective subcategory of $\mathbf{S}^{\mathbf{E}^{\text {op }}}$.

Proof This functor has a left adjoint given by $S:=\mathrm{id} \otimes S: \mathbf{S} \otimes \mathbf{S e t}^{\Xi^{\text {op }}} \rightarrow \mathbf{S} \otimes \mathbf{X}$. Moreover, id $\otimes N$ is clearly fully faithful.

Lemma 3.7 Let $\pi: \mathbf{R} \rightarrow \mathbf{S}$ be a surjective geometric morphism between Grothendieck toposes. The functor $\pi^{*} \otimes \mathrm{id}: \mathbf{S} \otimes \mathbf{X} \rightarrow \mathbf{R} \otimes \mathbf{X}$ creates colimits.

Proof A functor creates colimits if it is conservative and preserves colimits. The functor $\pi^{*} \otimes \mathrm{id}$ is a left adjoint and therefore preserves colimits. We claim that it is also conservative. Let $i$ be a map in $\mathbf{S} \otimes \mathbf{X}$ such that $\pi^{*} \otimes \operatorname{id}(i)$ is an isomorphism. Then $(\mathrm{id} \otimes N) \circ\left(\pi^{*} \otimes \mathrm{id}\right)(i)$ is an isomorphism. According to Proposition 3.4, we see that $\left(\pi^{*} \otimes \mathrm{id}\right) \circ(\mathrm{id} \otimes N)(i)$ is an isomorphism. By Proposition 3.6, the functor $\mathrm{id} \otimes N$ is conservative. Hence it is enough to prove the conservativity of $\pi^{*} \otimes$ id: $\mathbf{S}^{\Xi^{\text {op }}} \rightarrow \mathbf{R}^{\Xi^{\text {op }}}$, which follows immediately from the conservativity of $\pi^{*}$.

\section{Finite connected limit sketches}

Definition 4.1 [1, Definition 1.49] A limit sketch is a couple (T, $L)$ where $\mathbf{T}$ is a small category and $L$ is a collection of cones in $\mathbf{T}$.

Given a complete category $\mathbf{Y}$, a $\mathbf{Y}$-model for $(\mathbf{T}, L)$ is a functor $F: \mathbf{T} \rightarrow \mathbf{Y}$ sending all cones in $L$ to limit cones in $\mathbf{Y}$. The full subcategory of $\mathbf{Y}^{\mathbf{T}}$ spanned by the $\mathbf{Y}$-models for $(\mathbf{T}, L)$ will be denoted by $\operatorname{Mod}(\mathbf{T}, L)_{\mathbf{Y}}$. The category $\operatorname{Mod}(\mathbf{T}, L)_{\text {Set }}$ is simply denoted $\operatorname{Mod}(\mathbf{T}, L)$. It is a full reflective subcategory of $\mathbf{S e t}^{\mathbf{T}}$, and it is therefore locally presentable. Conversely, it can be shown that any locally presentable category is equivalent to the category of models of a limit sketch; see [1, Corollary 1.52].

For every locally presentable category $\mathbf{Y}$, the tensor product $\operatorname{Mod}(\mathbf{T}, L) \otimes \mathbf{Y}$ is equivalent to $\operatorname{Mod}(\mathbf{T}, L)_{\mathbf{Y}}$. In particular, for any locally presentable category $\mathbf{Y}$, the category $\operatorname{Mod}(\mathbf{T}, L)_{\mathbf{Y}}$ is locally presentable.

Definition 4.2 A finite connected limit sketch is a sketch $(\mathbf{T}, L)$ such that all cones in $L$ are indexed by finite connected diagrams.

The category $\operatorname{Mod}(\mathbf{T}, L)$ of Set-models for a finite connected sketch $(\mathbf{T}, L)$ is locally finitely presentable. Moreover, it is closed under coproducts and filtered colimits in Set $^{\mathrm{T}}$. In other words, the inclusion functor $i$ in the adjunction

$$
l: \operatorname{Set}^{\mathbf{T}} \rightleftarrows \operatorname{Mod}(\mathbf{T}, L): i
$$


preserves coproducts and filtered colimits. More broadly, we have the following lemma.

Lemma 4.3 Let $\mathbf{S}$ be a Grothendieck topos. In the adjunction

$$
l \otimes \mathrm{id}: \mathbf{S e t}^{\mathbf{T}} \otimes \mathbf{S} \rightleftarrows \mathbf{M o d}(\mathbf{T}, L) \otimes \mathbf{S}: i \otimes \mathrm{id},
$$

the right adjoint $i \otimes$ id preserves coproducts and filtered colimits.

Proof It is sufficient to prove that $\operatorname{Mod}(\mathbf{T}, L) \otimes \mathbf{S}$ is closed under coproducts and filtered colimits as a subcategory of $\mathbf{S}^{\mathbf{T}}$. This follows from the fact that in any Grothendieck topos, finite limits commute with filtered colimits and connected limits commute with coproducts.

The rest of this section will be devoted to the proof of the following proposition.

Proposition 4.4 Let $\mathbf{X}$ be a locally presentable category. The following are equivalent:

(1) There exists a small full subcategory $\Xi$ of $\mathbf{X}$ such that the pair $(\mathbf{X}, \Xi)$ satisfies Assumptions 1.1.

(2) There exists a finite connected limit sketch $(\mathbf{T}, L)$ and an equivalence of categories $\mathbf{X} \simeq \operatorname{Mod}(\mathbf{T}, L)$.

We first prove that (1) implies (2). We consider a cocomplete category $\mathbf{X}$ with a dense full subcategory $\Xi$ of compact and connected objects. We define $\widetilde{\Xi}$ to be the closure of $\Xi$ under finite connected colimits in $\mathbf{X}$. Then $\widetilde{\Xi}$ is dense and spanned by connected and compact objects; thus the nerve functor $\tilde{N}: \mathbf{X} \rightarrow \mathbf{S e t}^{\tilde{\Xi}^{\text {op }}}$ is fully faithful. Let $L$ be the set of representatives of all the finite connected limit cones in $\widetilde{\Xi}^{\text {op }}$; we then have the following result.

Lemma 4.5 The essential image of $\tilde{N}$ is the category of $\left(\widetilde{\Xi}^{\mathrm{op}}, L\right)-$ models.

Proof First, notice that for every $X \in \mathbf{X}$, the functor $\tilde{N}(X)$ sends all cones in $L$ to limit cones; indeed, if $D: I \rightarrow \widetilde{\Xi}$ is a finite connected diagram in $\widetilde{\Xi}$, then

$$
\tilde{N}(X)\left(\operatorname{colim}_{i \in I} D(i)\right) \cong \mathbf{X}\left(\operatorname{colim}_{i \in I} D(i), X\right) \cong \lim _{i \in I} \mathbf{X}(D(i), X) \cong \lim _{i \in I} \tilde{N}(X)(D(i)) \text {. }
$$

Thus it is sufficient to prove that every functor $F: \widetilde{\Xi}^{\text {op }} \rightarrow$ Set that preserves finite connected limits is in the essential image of $\tilde{N}$. The essential image of $\tilde{N}$ is closed under filtered colimits and coproducts; since the representables are all contained in the image of $\tilde{N}$, it is sufficient to show that $F$ is a coproduct of filtered colimits of 
representables. Since the category of representables is dense in $\mathbf{S e t}^{\tilde{\Xi}^{\text {op }}}$, it is sufficient to show that the connected components of the comma category $\widetilde{\Xi} \downarrow F$ are filtered.

This amounts to showing that, for every finite connected category $I$, every diagram $D: I \rightarrow \widetilde{\Xi} \downarrow F$ is the base of a cocone in $\widetilde{\Xi} \downarrow F$. Let $\pi: \widetilde{\Xi} \downarrow F \rightarrow \widetilde{\Xi}$ be the canonical projection. By the Yoneda lemma, giving the diagram $D$ is equivalent to giving a system of elements $\left\{x_{i} \in F(\pi D(i))\right\}_{i \in I}$ such that $F(f)\left(x_{j}\right)=x_{i}$ for every $f: i \rightarrow j$ in $I$. In other words, the data of the diagram $D$ is exactly the data of an element

$$
\left(x_{i}\right)_{i \in I} \in \lim _{i \in I} F(\pi D(i)) .
$$

Let $d \in \widetilde{\Xi}$ be the colimit of $\pi D$. By assumption,

$$
F(d) \cong \lim _{i \in I} F(\pi D(i))
$$

the $\left(x_{i}\right)_{i \in I}$ determines an arrow $d \rightarrow F$ in Set $^{\widetilde{\Xi}^{\text {op }}}$ which, seen as an object of $\widetilde{\Xi} \downarrow F$, is a vertex for a cocone over $D$.

Corollary 4.6 For every Grothendieck topos $\mathbf{S}$, the nerve functor

$$
N \otimes \mathrm{id}: \mathbf{X} \otimes \mathbf{S} \rightarrow \mathbf{S}^{\mathbf{\Xi}^{\text {op }}}
$$

preserves filtered colimits and coproducts.

Proof The functor $N \otimes$ id is isomorphic to the composite

$$
\mathbf{X} \otimes \mathbf{S} \stackrel{\tilde{N} \otimes \mathrm{id}}{\longrightarrow} \mathbf{S}^{\tilde{\Xi}^{\mathrm{op}}} \stackrel{l^{*}}{\longrightarrow} \mathbf{S}^{\Xi^{\mathrm{op}}}
$$

where $l: \Xi^{\text {op }} \rightarrow \widetilde{\Xi}^{\text {op }}$ is the canonical inclusion. The functor $\tilde{N} \otimes$ id preserves coproducts and filtered colimits by Lemma 4.3. The functor $l^{*}$ is left adjoint; hence it preserves all colimits.

We now want to prove that (2) implies (1) in Proposition 4.4. We start with a definition.

Definition 4.7 Let $(\mathbf{T}, L)$ be a limit sketch, we say that it is fully faithful if for any $t \in \mathbf{T}$, the functor $\mathbf{T}(t,-)$ is a model for $(\mathbf{T}, L)$.

Let $(\mathbf{T}, L)$ be a finite connected limit sketch, and let $\mathbf{X}$ be the category of its Setmodels. The inclusion $i: \mathbf{X} \rightarrow \mathbf{S e t}^{\mathbf{T}}$ has a left adjoint $l: \mathbf{S e t}^{\mathbf{T}} \rightarrow \mathbf{X}$.

Proposition 4.8 Assume that $(\mathbf{T}, L)$ is fully faithful. Then the category $\mathbf{T}^{\mathrm{op}}$, seen as a full subcategory of $\mathbf{X}$, is a dense subcategory whose objects are compact and connected. 
Proof The density of the inclusion $\mathbf{T}^{\mathrm{op}} \rightarrow \mathbf{X}$ is obvious. Let $t$ be in $\mathbf{T}$; we want to show that $h_{t}=\mathbf{T}(t,-)$ is connected and compact in $\mathbf{X}$. Let $F: D \rightarrow \mathbf{X}$ be a diagram; then we have

$$
\mathbf{X}\left(h_{t}, \operatorname{colim}_{d \in D} F(d)\right) \cong \operatorname{Set}^{\mathbf{T}}\left(h_{t}, i\left(\operatorname{colim}_{d \in D} F(d)\right)\right) .
$$

Hence if $D$ is a filtered category or a discrete category, we have

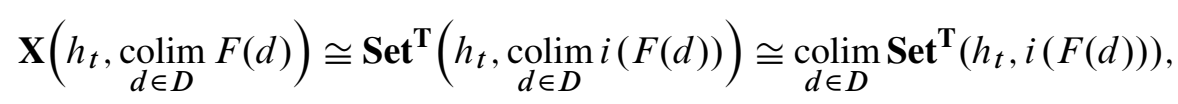

where the second equality follows from the fact that colimits are computed objectwise in a presheaf category. It follows that the object $h_{t} \in \mathbf{X}$ is compact and connected.

Now, let $(\mathbf{T}, L)$ be a general finite connected limit sketch. Let $\mathbf{X}$ be the category of its Set-models. Let $\mathbf{A}=l \circ y\left(\mathbf{T}^{\mathrm{op}}\right)$ be the image of $l$ composed with the Yoneda embedding. The category $\mathbf{A}$ is dense in $\mathbf{X}$; moreover, the objects of $\mathbf{A}$ are compact and connected in $\mathbf{X}$ since $i$ preserves filtered colimits and coproducts. Let $\theta: \mathbf{T} \rightarrow \mathbf{A}^{\mathrm{op}}$ be the map induced by restricting $(l \circ y)^{\mathrm{op}}$. There is an induced adjunction

$$
\operatorname{Set}^{\mathbf{T}} \underset{\theta_{*}}{\stackrel{\theta^{*}}{\rightleftarrows}} \operatorname{Set}^{\mathbf{A}^{\mathrm{op}}} \text {. }
$$

We can then consider the finite connected limit sketch $\left(\mathbf{A}^{\mathrm{op}}, \theta(L)\right)$. The following proposition shows that it is fully faithful and that its category of models is equivalent to $\mathbf{X}$, which according to Proposition 4.8, will conclude the proof of Proposition 4.4.

Proposition 4.9 The adjunction $\left(\theta^{*}, \theta_{*}\right)$ restricts to an equivalence of categories

$$
\operatorname{Mod}(\mathbf{T}, L)_{\text {Set }} \underset{\theta_{*}}{\stackrel{\theta^{*}}{\rightleftarrows}} \operatorname{Mod}\left(\mathbf{A}^{\mathrm{op}}, \theta(L)\right)_{\text {Set }}
$$

Proof For every $t \in \mathbf{T}$, we denote the representable functor $\mathbf{T}(t,-)$ by $h_{t}$. For every $a \in \mathbf{A}$, the functor $i(a) \in \mathbf{S e t}^{\mathbf{T}}$ is isomorphic to

$$
\operatorname{colim}_{\{t \rightarrow i(a)\} \in \mathbf{T}^{\mathrm{To}} \downarrow i(a)} h_{t},
$$

and by adjunction, the indexing category $\mathbf{T}^{\mathrm{op}} \downarrow i(a)$ is isomorphic to $\theta^{\mathrm{op}} \downarrow a$.

Suppose that $G \in \mathbf{S e t}^{\mathbf{T}}$ belongs to $\operatorname{Mod}(\mathbf{T}, L)_{\text {Set }}$. For every $a \in \mathbf{A}^{\text {op }}$, the right Kan extension $\theta_{*}(G)$ of $G$ along $\theta$, has $a$-component

$$
\begin{aligned}
\theta_{*} G(a) \cong \lim _{\{a \rightarrow \theta(t)\} \in a \downarrow \theta} G(t) & \cong \operatorname{Set}^{\mathbf{T}}\left(\underset{\{\theta(t) \rightarrow a\} \in \theta^{\circ \mathrm{o} \downarrow} \downarrow}{\left.\operatorname{colim}_{t} h_{t}, G\right)}\right. \\
& \cong \operatorname{Set}^{\mathbf{T}}(i(a), G) \cong \operatorname{Mod}(\mathbf{T}, L)(a, G) .
\end{aligned}
$$


It follows that for every $t \in \mathbf{T}$, we have

$$
\theta^{*} \theta_{*} G(t) \cong \operatorname{Mod}(\mathbf{T}, L)(l(t), G) \cong \operatorname{Set}^{\mathbf{T}}(y(t), G) \cong G(t) .
$$

This implies that the counit $\varepsilon: \theta^{*} \theta_{*}(G) \rightarrow G$ is an isomorphism. Since, by assumption, $G$ belongs to $\operatorname{Mod}(\mathbf{T}, L)_{\text {Set }}$, this also implies that $\theta_{*} G$ belongs to $\operatorname{Mod}(\mathbf{A}, \theta(L))_{\text {Set }}$. Note that for every $a \in \mathbf{A}$, the representable functor $h_{a} \in \mathbf{S e t}^{\mathbf{t}^{\mathrm{op}}}$ is a model for $(\mathbf{A}, \theta(L))$. Conversely, if $F \in \mathbf{S e t}^{\mathbf{A}^{\mathrm{op}}}$ is a model for $(\mathbf{A}, \theta(L))$, then $\theta^{*} F$ is a model for (T, $L)$; furthermore, the unit $\eta: F \rightarrow \theta_{*} \theta^{*}(F)$ is an isomorphism. In fact, for every $a \in \mathbf{A}^{\mathrm{op}}$,

$$
F(a) \cong \operatorname{Set}^{\mathbf{T}}\left(i(a), \theta^{*}(F)\right) \cong \operatorname{Set}^{\mathbf{T}}\left(\underset{\{\theta(t) \rightarrow a\} \in \theta^{\mathrm{op} \downarrow} \downarrow a}{\operatorname{colim}} h_{t}, \theta^{*} F\right) \cong \theta_{*} \theta^{*} F(a) .
$$

\section{Construction of the model structure}

In all this section, we fix a pair $(\mathbf{X}, \Xi)$ satisfying Assumptions 1.1. For $\mathbf{Y}$ and $\mathbf{Z}$ two locally presentable categories, we denote by $(Y, Z) \mapsto Y \otimes Z$ the canonical functor $\mathbf{Y} \times \mathbf{Z} \rightarrow \mathbf{Y} \otimes \mathbf{Z}$.

Lemma 5.1 Let $\mathbf{S}$ be a Grothendieck topos. Let $C$ be an object in $\mathbf{X}$ and let $i: K \rightarrow L$ be a monomorphism in $\mathbf{S}$. If

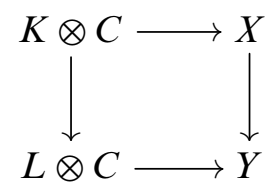

is a pushout diagram in $\mathbf{S} \otimes \mathbf{X}$, then its nerve is a pushout diagram in $\mathbf{S}^{\Xi^{\text {op }}} \cong \mathbf{S} \otimes \mathbf{S e t}^{\Xi^{\text {op }}}$.

Proof Let us denote by $\square$ the above square. By [16, Theorem 7.54], there exists a Boolean topos $\widetilde{\mathbf{S}}$ with a surjective geometric morphism $\pi: \widetilde{\mathbf{S}} \rightarrow \mathbf{S}$. Recall from Proposition 3.4 that the square

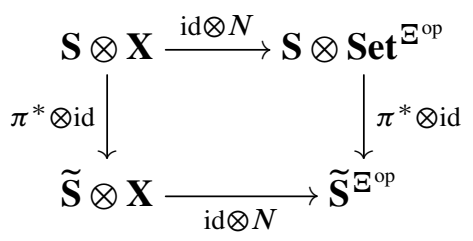

commutes up to natural isomorphism. Moreover, the vertical maps create colimits.

The square $\square$ lives in the upper left corner. We want to prove that $\operatorname{id} \otimes N(\square)$ is a pushout square. By Lemma 3.7, we know that $\pi^{*} \otimes$ id creates colimits. Hence id $\otimes N(\square)$ is a 
pushout if and only if ( $\left.\pi^{*} \otimes \mathrm{id}\right) \circ(\mathrm{id} \otimes N)(\square)$ is a pushout square. By commutativity of the above diagram, (id $\otimes N)(\square)$ is a pushout if and only if (id $\otimes N) \circ\left(\pi^{*} \otimes \mathrm{id}\right)(\square)$ is a pushout. We know by assumption that $\square$ is a pushout; therefore, since $\pi^{*} \otimes$ id is a left adjoint, $\left(\pi^{*} \otimes \mathrm{id}\right)(\square)$ is a pushout. Hence, we want to prove that (id $\left.\otimes N\right)(\square)$ is a pushout knowing that (id $\otimes N)\left(\pi^{*} \otimes \mathrm{id}\right)(\square)$ is a pushout. In other words, we are reduced to proving the lemma in the case where $\mathbf{S}=\widetilde{\mathbf{S}}$ is a Boolean topos.

Using Remark 3.5, we are reduced to proving that if $K \rightarrow L$ is a monomorphism in $\widetilde{\mathbf{S}}$, and the square

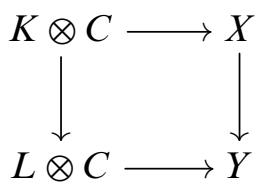

is a pushout square in $\widetilde{\mathbf{S}} \otimes \mathbf{X}$, then the square

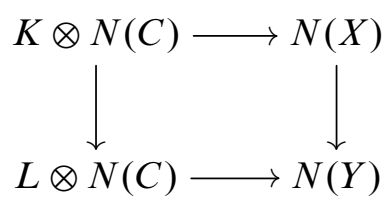

is a pushout square in $\widetilde{\mathbf{S}}^{\Xi^{\text {op }}}$. Since $\widetilde{\mathbf{S}}$ is Boolean, we can write $L=K \sqcup(L-K)$, where $L-K$ denotes a complement of $K$. Hence, we know that $Y=(L-K) \otimes C \sqcup X$. Since $N$ commutes with coproducts (see Corollary 4.6), we have

$$
N(Y)=N(X) \sqcup N((L-K) \otimes C) \cong L \otimes N(C) \sqcup_{K \otimes N(C)} N(X)
$$

as desired.

Now, $\mathbf{S}$ denotes a Grothendieck topos possessing a model structure that satisfies Assumption 3.2. We let $I$ (resp. $J$ ) be a set of generating (trivial) cofibrations for $\mathbf{S}$. The category $\mathbf{S}^{\mathbf{\Xi}^{\text {op }}}$ can then be equipped with a projective model structure in which the weak equivalences (resp. fibrations) are the maps that are objectwise weak equivalences (resp. fibrations) in $\mathbf{S}$. We denote by $y: \Xi \rightarrow \mathbf{S e t}^{\boldsymbol{\Xi}^{\text {op }}}$ the Yoneda embedding. Then a set of generating cofibrations (resp. trivial cofibrations) for the projective model structure on $\mathbf{S}^{\mathbf{E}^{\text {op }}} \cong \mathbf{S} \otimes \mathbf{S e t}^{\mathbf{E}^{\text {op }}}$ is

$$
\mathcal{J}=\{i \otimes y(\xi) \mid i \in I, \xi \in \Xi\} \quad(\text { resp. } \mathcal{J}=\{j \otimes y(\xi) \mid j \in J, \xi \in \Xi\}) .
$$

We then have the following proposition.

Proposition 5.2 The functor id $\otimes N: \mathbf{S} \otimes \mathbf{X} \rightarrow \mathbf{S}^{\Xi^{\text {op }}}$ sends pushouts of maps in $(\mathrm{id} \otimes S)(\mathcal{J})$ and $(\mathrm{id} \otimes S)(\mathcal{J})$ to J-cofibrations and $\mathcal{J}$-cofibrations, respectively. 
Proof Let $i \otimes y(\xi) \in \mathcal{J}$ (resp. $i \otimes y(\xi) \in \mathcal{J})$. Then $(\mathrm{id} \otimes S)(i \otimes y(\xi)) \cong i \otimes \xi$. By Lemma 5.1, id $\otimes N$ preserves pushout along such maps; since $(\mathrm{id} \otimes N)(i \otimes \xi) \cong$ $i \otimes y(\xi) \in \mathcal{J}$ (resp. J), the statement follows.

Proposition 5.3 There is a model structure on $\mathbf{S} \otimes \mathbf{X}$ whose weak equivalences and fibrations are created by the nerve functor (id $\otimes N$ ): $\mathbf{S} \otimes \mathbf{X} \rightarrow \mathbf{S}^{\Xi^{\text {op }}}$. The adjunction $(\mathrm{id} \otimes S, \mathrm{id} \otimes N$ ) becomes a Quillen adjunction with respect to this model structure. Moreover, the nerve functor preserves cofibrations.

Proof We transfer the projective model structure on $\mathbf{S}^{\Xi^{\text {op }}}$ to $\mathbf{S} \otimes \mathbf{X}$ using the adjunction $(\mathrm{id} \otimes S, \mathrm{id} \otimes N)$. Since all objects of $\Xi$ are compact, $\mathrm{id} \otimes N$ preserves filtered colimits. The statement is an application of [14, Theorem 1.2] using Proposition 5.2.

Proposition 5.4 Suppose that the model structure on $\mathbf{S}$ is left (resp. right) proper. Then the model structure on $\mathbf{S} \otimes \mathbf{X}$ of Proposition 5.3 is left (resp. right) proper.

Proof Since $(\mathrm{id} \otimes N)$ preserves pullbacks and fibrations and reflects weak equivalences, the fact that $\mathbf{S} \otimes \mathbf{X}$ is right proper if $\mathbf{S}$ is right proper follows directly from the fact that the projective model structure on $\mathbf{S}^{\Xi^{\text {op }}}$ is right proper.

Suppose now that $\mathbf{S}$ is left proper. In order to prove that $\mathbf{S} \otimes \mathbf{X}$ is left proper, we have to prove that for every cofibration $s: A \rightarrow B$ and every weak equivalence $w: A \rightarrow X$, the map $w^{\prime}$ in the pushout square

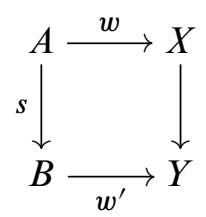

is again a weak equivalence. Since every cofibration is the retract of a relative $(\mathrm{id} \otimes S)(\mathcal{J})$-cell complex, it is sufficient to consider the case in which $s$ is a relative $(\mathrm{id} \otimes S)(\mathcal{J})$-cell complex. Since (id $\otimes N)$ reflects weak equivalences, it is sufficient to check that $(\mathrm{id} \otimes N)\left(w^{\prime}\right)$ is a weak equivalence.

We now prove that $(\mathrm{id} \otimes N)$ sends pushouts along relative $(\mathrm{id} \otimes S)(\mathcal{J})$-cell complexes to pushout squares. The statement will follow by applying (id $\otimes N$ ) to (5-1) since $(\mathrm{id} \otimes N)$ preserves cofibrations by Proposition 5.3 and the projective model structure on $\mathbf{S}^{\mathbf{E}^{\text {op }}}$ is left proper.

We are left to prove that $(\mathrm{id} \otimes N)$ sends pushouts along a relative (id $\otimes S)(\mathcal{J})$-cell complex $s$ to pushout squares. Note that $(\mathrm{id} \otimes N)$ preserves filtered colimits; thus it is 
sufficient to prove our claim in the case in which $s$ is the pushout of a map $i \otimes \xi \in$ $(\mathrm{id} \otimes S)(\mathcal{J})$ for some $i \in I$ and $\xi \in \Xi$. In this last case, we have a commutative diagram

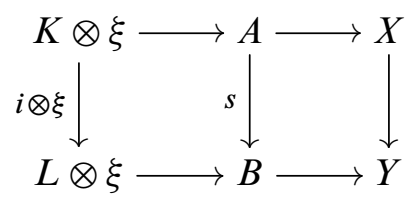

of pushout squares, and we have to prove that (id $\otimes N$ ) sends the right square to a pushout square. By Lemma 5.1 the left square and the external square are both sent to pushout squares by (id $\otimes N$ ); it follows that the right square is sent to a pushout square as well.

We now prove that the Quillen pair (id $\otimes S, \mathrm{id} \otimes N$ ) of Proposition 5.3 is a Quillen equivalence. This will follow from the next proposition.

Proposition 5.5 Let $f: X \rightarrow Y$ be a cofibration in $\mathbf{S}^{\Xi^{\text {op }}}$. Assume the unit map $X \rightarrow$ $(\mathrm{id} \otimes N)(\mathrm{id} \otimes S)(X)$ is an isomorphism. Then the unit map $Y \rightarrow(\mathrm{id} \otimes N)(\mathrm{id} \otimes S)(Y)$ is an isomorphism.

Proof Since isomorphisms are closed under retracts, and cofibrations in $\mathbf{S}^{\mathbf{\Xi}^{\text {op }}}$ are retracts of J-cells complexes, we can assume, without loss of generality, that $f$ is an J-cell complex. Thus suppose that $f$ is the transfinite composition indexed by an ordinal $\beta$,

$$
X \cong X_{0} \rightarrow X_{1} \rightarrow \cdots \rightarrow \operatorname{colim}_{\alpha<\beta} X_{\alpha} \cong Y=: X_{\beta},
$$

in which every map is a pushout along a map of the kind $i \otimes \xi$ for some $i \in I$ and $\xi \in \Xi$. Now, we prove by transfinite induction that for each $\alpha$, the unit map $X_{\alpha} \rightarrow(\mathrm{id} \otimes N)(\mathrm{id} \otimes S)\left(X_{\alpha}\right)$ is an isomorphism. We already know that the unit map $X \rightarrow(\mathrm{id} \otimes N)(\mathrm{id} \otimes S)(X)$ is an isomorphism. Assume that the result holds for $X_{\alpha}$. We have the diagram

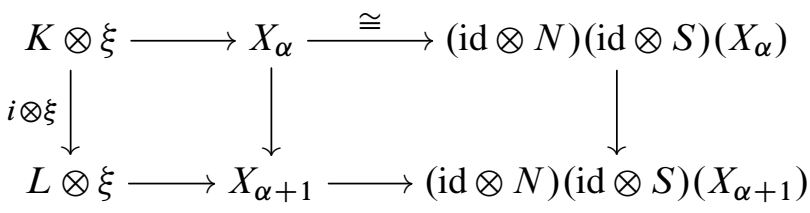

in which the left square is a pushout square, and the external square is also a pushout square by Lemma 5.1; it follows that the right square is also a pushout square, and therefore, the unit $X_{\alpha+1} \rightarrow(\mathrm{id} \otimes N)(\mathrm{id} \otimes S)\left(X_{\alpha+1}\right)$ is an isomorphism. 
Finally, if $\alpha \leq \beta$ is a limit ordinal and the result holds for $X_{\alpha^{\prime}}$ for any $\alpha^{\prime}<\alpha$, then it holds for $X_{\alpha} \cong \operatorname{colim}_{\alpha^{\prime}<\alpha} X_{\alpha^{\prime}}$ as well since a filtered colimit of isomorphisms is an isomorphism.

Corollary 5.6 If $X$ is a cofibrant object of $\mathbf{S}^{\Xi^{\text {op }}}$, then the unit map $X \rightarrow(\mathrm{id} \otimes N) \circ$ $(\mathrm{id} \otimes S)(X)$ is an isomorphism.

Proof This is just Proposition 5.5 applied to the map $\varnothing \rightarrow X$, observing that both $(\mathrm{id} \otimes S)$ and $(\mathrm{id} \otimes N)$ preserve the initial object (this uses the fact that the objects of $\Xi$ are connected in $\mathbf{X}$ ).

Proposition 5.7 The Quillen adjunction of Proposition 5.3,

$$
(\mathrm{id} \otimes S): \mathbf{S}^{\Xi^{\text {op }}} \leftrightarrows \mathbf{S} \otimes \mathbf{X}:(\operatorname{id} \otimes N)
$$

is a Quillen equivalence.

Proof Let $f:(\operatorname{id} \otimes S)(X) \rightarrow Y$ be a map in $\mathbf{S} \otimes \mathbf{X}$ where $X$ is a cofibrant object in $\mathbf{S}^{\mathbf{\Xi}^{\text {op }}}$ and $Y$ is a fibrant object in $\mathbf{S} \otimes \mathbf{X}$; we have to prove that $f$ is a weak equivalence if and only if the composition of (id $\otimes N)(f)$ with the unit map of the adjunction $X \rightarrow(\mathrm{id} \otimes N)(\mathrm{id} \otimes S)(X) \rightarrow(\mathrm{id} \otimes N)(Y)$ is a weak equivalence. Since the unit map is an isomorphism by Corollary 5.6 and the functor (id $\otimes N$ ) reflects and preserves weak equivalences, the statement follows from the two-out-of-three property for weak equivalences.

We have also the following characterization of the cofibrations.

Proposition 5.8 A map $f: A \rightarrow B$ in $\mathbf{S} \otimes \mathbf{X}$ is a cofibration if and only if (id $\otimes N)(f)$ is a projective cofibration.

Proof We know that (id $\otimes N)$ preserves cofibrations. Conversely, if (id $\otimes N)(f)$ is a cofibration, then (id $\otimes S) \circ(\mathrm{id} \otimes N)(f)$ is a cofibration. But since the functor (id $\otimes S) \circ(\mathrm{id} \otimes N)$ is isomorphic to the identity functor, we are done.

We can now state and prove our main theorem.

Theorem 5.9 Assume that $\mathbf{S}$ is left proper and that $\mathbf{S}^{\Xi^{\text {op }}}$ is equipped with a model structure that is a left Bousfield localization of the projective model structure with respect to a set of maps. Then there is a model structure on $\mathbf{S} \otimes \mathbf{X}$ in which a map is a fibration (resp. cofibration, weak equivalence) if and only if its image under id $\otimes N$ is a fibration (resp. cofibration, weak equivalence). The functor id $\otimes N$ is then a right Quillen equivalence. Finally, the model structure on $\mathbf{S} \otimes \mathbf{X}$ is left proper, and it is right proper if $\mathbf{S}^{\Xi^{\text {op }}}$ is right proper. 
Proof We denote by $\mathbf{S}_{\text {proj }}^{\Xi^{\text {op }}}$ the projective model structure on $\mathbf{S}^{\Xi^{\text {op }}}$ and by $\mathbf{S} \otimes \mathbf{X}_{\text {proj }}$ the model structure constructed in Proposition 5.3. By assumption, the model structure $\mathbf{S}^{\mathbf{\Xi}^{\text {op }}}$ is obtained by localizing $\mathbf{S}_{\text {proj }}^{\Xi^{\text {op }}}$ with respect to a set $U$ of maps in $\mathbf{S}^{\Xi^{\text {op }}}$. Let $V$ be the set of maps of $\mathbf{S} \otimes \mathbf{X}$ of the form $\mathbb{L}(\mathrm{id} \otimes S)(u)$ for $u \in U$. Since $\mathbf{S} \otimes \mathbf{X}_{\text {proj }}$ is left proper (by Proposition 5.4) and combinatorial, the left Bousfield localization $L_{V}\left(\mathbf{S} \otimes \mathbf{X}_{\text {proj }}\right)$ of $\mathbf{S} \otimes \mathbf{X}_{\text {proj }}$ exists.

According to [13, Theorem 3.3.20] and Proposition 5.7, there is a Quillen equivalence

$$
(\mathrm{id} \otimes S): L_{U}\left(\mathbf{S}_{\text {proj }}^{\Xi^{\mathrm{op}}}\right) \leftrightarrows L_{V}\left(\mathbf{S} \otimes \mathbf{X}_{\text {proj }}\right):(\mathrm{id} \otimes N)
$$

where the right adjoint preserves and reflects weak equivalences by [14, Proposition 3.7]. Cofibrations are not changed by a left Bousfield localization; hence, by Proposition 5.8, a map is a cofibration if and only if its image under ( $\mathrm{id} \otimes N$ ) is one. It remains to show that id $\otimes N$ reflects fibrations. Let $p: U \rightarrow V$ be a map in $L_{V}\left(\mathbf{S} \otimes \mathbf{X}_{\text {proj }}\right)$ that is sent to a fibration by (id $\otimes N)$. Let $i: A \rightarrow B$ be a trivial cofibration in $L_{V}\left(\mathbf{S} \otimes \mathbf{X}_{\text {proj }}\right)$. Let

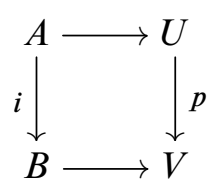

be a commutative diagram. We want to produce a lift. We can hit this diagram with $\mathrm{id} \otimes N$. The map id $\otimes N(i)$ is a trivial cofibration. It follows that there exists a map $l: \mathrm{id} \otimes N(B) \rightarrow \mathrm{id} \otimes N(U)$ making the diagram

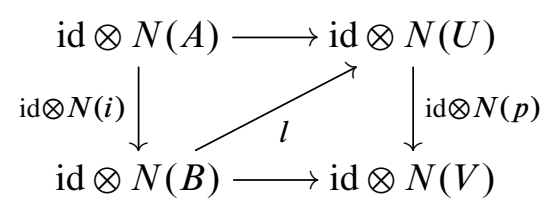

commute. Since id $\otimes N$ is fully faithful, this implies the existence of a lift for the initial square.

Finally, the model structure is left proper, as is any left Bousfield localization of a left proper model structure, and the argument for right properness is similar to the one in Proposition 5.4.

\section{Enrichment}

Let $\mathbf{V}$ be a cofibrantly generated monoidal model category. In this section, we study the existence of the structure of a $\mathbf{V}$-enriched model category on the model categories of Theorem 5.9. 
Let $\mathbf{C}$ be a bicomplete $\mathbf{V}$-enriched category that we assume to be tensored and cotensored over $\mathbf{V}$. For every $C \in \mathbf{C}$ and $V \in \mathbf{V}$, we denote by $V \odot C$ and $C^{V}$ the tensor of $C$ with $V$ and the cotensor of $C$ with $V$, respectively, while the hom-functor will be denoted by $\operatorname{Hom}_{\mathbf{V}}(-,-)$. Recall that $\mathbf{C}$ is a $\mathbf{V}$-enriched model category if it has a model structure such that, for every cofibration $i: K \rightarrow L$ in $\mathbf{V}$ and every cofibration $j: A \rightarrow B$ in $\mathbf{C}$, the induced pushout-product map

$$
i \square j: L \odot A \underset{K \odot A}{\sqcup} K \odot B \longrightarrow L \odot B
$$

is a cofibration in $\mathbf{C}$ which is, moreover, a weak equivalence if either $i$ or $j$ are trivial cofibrations.

For every small category $D$, the category $\operatorname{Fun}(D, \mathbf{C})$ is also $\mathbf{V}$-enriched and bicomplete in a natural way; see [12, Section 4.1]. We now suppose that our category of "spaces" $\mathbf{S}$, satisfying Assumption 3.2, is enriched over $\mathbf{V}$, with an enrichment that gives it the structure of a $\mathbf{V}$-model category; in particular, $\mathbf{S}$ is tensored and cotensored over $\mathbf{V}$. For every locally presentable category $\mathbf{Y}$ that we can suppose isomorphic to $\operatorname{lnd}_{\lambda}(A)$ for some small category $A$, the category $\mathbf{Y} \otimes \mathbf{S}$, seen as a full subcategory of $\operatorname{Fun}\left(A^{\mathrm{op}}, \mathbf{S}\right)$, is naturally enriched in $\mathbf{V}$. It is also bicomplete as a $\mathbf{V}$-category. For every $V \in \mathbf{V}$, the tensor with $V$ is given by $\operatorname{id} \otimes(V \odot-)$ and the cotensor with $V$ is given by $\operatorname{id} \otimes\left(-{ }^{V}\right)$. Given another locally presentable category $\mathbf{Z}$, every adjunction $u^{*}: \mathbf{Y} \rightleftarrows \mathbf{Z}: u_{*}$ induces a $\mathbf{V}$-adjunction

$$
u^{*} \otimes \mathrm{id}: \mathbf{Y} \otimes \mathbf{S} \rightleftarrows \mathbf{Z} \otimes \mathbf{S}: u_{*} \otimes \mathrm{id} .
$$

Indeed, $u_{*} \otimes$ id extends to a $\mathbf{V}$-functor, and it preserves cotensors; thus it has a $\mathbf{V}$-left adjoint by [17, Theorem 4.85$]$.

The following proposition can be seen as a particular case of [12, Theorem 1.16].

Proposition 6.1 Let $\mathbf{V}$ be a cofibrantly generated monoidal model category, and suppose that $\mathbf{S}$ is $\mathbf{V}$-enriched as a model category. Then the model structure on $\mathbf{S} \otimes \mathbf{X}$ of Proposition 5.3 is $\mathbf{V}$-enriched.

Proof Recall that if $\mathbf{S}$ is a $\mathbf{V}$-model category, then $\mathbf{S}^{\mathbf{\Xi}^{\text {op }}}$ is naturally $\mathbf{V}$-enriched, and the projective model structure is a $\mathbf{V}$-model structure; see [12, 4.4]. The adjunction (id $\otimes S$, id $\otimes N$ ) can be extended to a $\mathbf{V}$-adjunction; since id $\otimes N$ is right adjoint, it preserves cotensors.

To prove that $\mathbf{S} \otimes \mathbf{X}$ is a $\mathbf{V}$-model structure, it is sufficient to check that for every cofibration $i: K \rightarrow L$ in $\mathbf{V}$ and every fibration in $i: X \rightarrow Y$ in $\mathbf{S} \otimes \mathbf{X}$, the morphism

$$
\left(i^{*}\right)^{p_{*}}: L^{X} \longrightarrow Y^{L} \underset{K^{Y}}{\times} K^{X}
$$


is a fibration, and a weak equivalence if $i$ or $p$ are weak equivalences; see [12, 4.3]. Since id $\otimes N$ creates (trivial) fibrations and preserves cotensors, the above statement follows from the fact that the projective model structure on $\mathbf{S}^{\Xi^{\text {op }}}$ is $\mathbf{V}$-enriched.

Following [2], we call a model category tractable if it is combinatorial and admits a set of generating cofibrations with cofibrant source. Let us assume that $\mathbf{V}$ is a tractable symmetric monoidal model category. We recall that, given a $\mathbf{V}$-model structure $\mathbf{C}$ and a class of maps $H$ in $\mathbf{C}$, the $\mathbf{V}$-enriched left Bousfield localization of $\mathbf{C}$ at $H$, denoted by $L_{H / \mathbf{V}}(\mathbf{C})$, is the unique $\mathbf{V}$-enriched model structure on (the underlying $\mathbf{V}$-category of) $\mathbf{C}$ (if it exists) such that:

- The cofibrations are the cofibrations of $\mathbf{C}$.

- An object $F$ is fibrant in $L_{H / \mathbf{V}}(\mathbf{C})$ if and only if it is fibrant in $\mathbf{C}$, and the map

$$
h_{*}: \mathbb{R} \operatorname{Hom}_{\mathbf{V}}(B, F) \rightarrow \mathbb{R} \operatorname{Hom}_{\mathbf{V}}(A, F)
$$

is a weak equivalence (in $\mathbf{V}$ ) for every $h \in H$.

Barwick showed in [2, Theorem 4.46] that if $\mathbf{C}$ is a tractable left proper $\mathbf{V}$-model category and $H$ is a set of maps, the $\mathbf{V}$-enriched left Bousfield localization $L_{H / \mathbf{V}}(\mathbf{C})$ always exists (and it is tractable).

Remark 6.2 When $\mathbf{V}$ is the category $\operatorname{Set}^{\Delta^{\mathrm{op}}}$ with the Kan-Quillen model structure, $\mathbf{V}$-enriched Bousfield localization at $H$ coincides with the usual Bousfield localization at $H$, and it is enough to assume that $\mathbf{C}$ is combinatorial and left proper for $L_{H / \text { Set }^{\Delta^{\text {op }}}(\mathbf{C})}$ to exist; see [13, Theorem 4.1.1].

Now assume that our category of spaces $\mathbf{S}$ is a model category satisfying Assumption 3.2 which is furthermore a left proper tractable $\mathbf{V}$-model category. Under these assumptions, $\mathbf{S}_{\text {proj }}^{\Xi_{\text {op }}}$ is also tractable and left proper, and the same holds for the model structure $\mathbf{S} \otimes \mathbf{X}_{\text {proj }}$ of Proposition 5.3. The following is an enriched version of Theorem 5.9.

Proposition 6.3 Suppose $H$ is a set of maps in $\mathbf{S}^{\Xi^{\text {op }}}$. The $\mathbf{V}$-model structure on $L_{H / \mathbf{V}}\left(\mathbf{S}^{\Xi^{\text {op }}}\right)$ can be transferred along id $\otimes N$ to a $\mathbf{V}$-enriched model structure on $\mathbf{S} \otimes \mathbf{X}$ in which a map is a fibration (resp. cofibration, weak equivalence) if and only if its image by id $\otimes N$ is a fibration (resp. cofibration, weak equivalence). The functor $\mathrm{id} \otimes N$ is then a right Quillen equivalence. Finally, the model structure on $\mathbf{S} \otimes \mathbf{X}$ is left proper, and it is right proper if $\mathbf{S}$ is right proper. 
Proof The enriched left Bousfield localization $L_{H / \mathbf{V}}\left(\mathbf{S}^{\Xi^{\text {op }}}\right)$ is the usual Bousfield localization of the projective model structure on $\mathbf{S}^{\mathbf{\Xi}^{\text {op }}}$ with respect to

$$
H \square I=\{h \square i \mid h \in H, i \in I\},
$$

where $I$ is a set of generating cofibrations for $\mathbf{V}$ [2, Theorem 4.46]; the statement follows from Theorem 5.9. The transfer of this model structure on $\mathbf{S} \otimes \mathbf{X}$ is the localization of $\mathbf{S} \otimes \mathbf{X}_{\text {proj }}$ with respect to $\mathbb{L}(\mathrm{id} \otimes S)(H \square I)=\mathbb{L}(\mathrm{id} \otimes S)(H) \square I$, and it is thus the $\mathbf{V}$-enriched Bousfield localization $L_{\mathbb{L}(\mathrm{id} \otimes S) H / \mathbf{V}}\left(\mathbf{S}^{\Xi^{\mathrm{op}}}\right)$.

\section{Applications}

In this last section, we study some of the consequences of Theorem 5.9. In the first subsection, we compare strict models for a fully faithful (see Definition 4.7) finite connected limit sketch to homotopy models. Then we describe the consequences of this comparison to the theories of operads, $n$-categories and $n$-fold categories. Finally, in the last subsection, we explain how our work relates to the theory of monads with arities of [5].

\subsection{Models for a finite connected limit sketch}

Let $(\mathbf{T}, L)$ be a finite connected limit sketch, and let $\left(\mathbf{A}^{\mathrm{op}}, \theta(L)\right)$ be the finite connected fully faithful sketch of Proposition 4.9. Note that if (T, $L)$ is already fully faithful, then $\left(\mathbf{A}^{\mathrm{op}}, \theta(L)\right)$ is isomorphic to $(\mathbf{T}, L)$. Let $(S, N)$ be the nerve adjunction for $\mathbf{A}$, seen as a dense full subcategory of $\operatorname{Mod}(\mathbf{T}, L)$.

Let $\mathbf{S}$ be a model category satisfying Assumption 3.2. We have the category $\operatorname{Mod}(\mathbf{T}, L)_{\mathbf{S}}$ of strict models for (T, $L)$ that are internal to $\mathbf{S}$, and the adjunction (4-1) decomposes as

$$
\mathbf{S}^{\mathbf{T}} \underset{\theta^{*}}{\stackrel{\theta_{!}}{\rightleftarrows}} \mathbf{S}^{\mathbf{A}^{\mathrm{op}}} \underset{N \otimes \mathrm{id}}{\stackrel{S \otimes \mathrm{id}}{\rightleftarrows}} \operatorname{Mod}(\mathbf{T}, L)_{\mathbf{S}}
$$

We then have the following proposition.

Proposition 7.1 The projective model structure on $\mathbf{S}^{\mathbf{T}}$ can be transferred along the nerve functor

$$
i \otimes \mathrm{id}: \operatorname{Mod}(\mathbf{T}, L)_{\mathbf{S}} \rightarrow \mathbf{S}^{\mathbf{T}} .
$$

Moreover, if $(\mathbf{T}, L)$ is a fully faithful sketch, the functor $N \otimes$ id becomes a Quillen equivalence. 
Proof Since the functor $\theta: \mathbf{T} \rightarrow \mathbf{A}^{\mathrm{op}}$ is a bijection on objects, the projective model structure on $\mathbf{S}^{\mathbf{T}}$ transfers along $\theta^{*}$ to the projective model structure on $\mathbf{S}^{\mathbf{A}^{\text {op }}}$. Since $\mathbf{A}$ is a dense full subcategory of $\operatorname{Mod}(\mathbf{T}, L)$, the projective model structure transfers along $N \otimes$ id by Theorem 5.9, and $N \otimes$ id is a Quillen equivalence. It follows that the projective model structure on $\mathbf{S}^{\mathbf{T}}$ transfers along $l \otimes$ id. If (T, $\left.L\right)$ is a fully faithful sketch, $\mathbf{T}$ is isomorphic to $\mathbf{A}^{\mathrm{op}}$; thus $l \otimes \mathrm{id}$ is a Quillen equivalence.

Now, we want to localize the model structure on $\mathbf{S}^{\mathbf{T}}$ to obtain a new one in which the fibrant objects are suitable notions of homotopy models for $(\mathbf{T}, L)$. Roughly speaking, a homotopy model for a limit sketch $(\mathbf{T}, L)$ is a diagram $\mathbf{T} \rightarrow \mathbf{S}$ that sends the cones of $L$ to homotopy limit cones. For a model category $\mathbf{M}$, we denote by $\mathbb{R} \operatorname{Map}_{\mathbf{M}}(X, Y)$ the derived mapping space from $X$ to $Y$. If $\mathbf{M}$ is a simplicial model category, this is just given by the ordinary mapping space from a cofibrant replacement of $X$ to a fibrant replacement of $Y$. In general, this can be constructed as the mapping space in the Hammock localization or via simplicial/cosimplicial framings.

Lemma 7.2 Let $\mathbf{M}$ be a combinatorial model category. There exists a set $\mathcal{G}$ of objects of $\mathbf{M}$ with the following property: a map $f: X \rightarrow Y$ is a weak equivalence of $\mathbf{M}$ if and only if the induced map

$$
\mathbb{R} \operatorname{Map}_{\mathbf{M}}(G, X) \rightarrow \mathbb{R} \operatorname{Map}_{\mathbf{M}}(G, Y)
$$

is a weak equivalence for any $G$ in $\mathcal{G}$.

Proof By a result of Dugger (see [11, Propositions 3.2, 3.3]), there exists a small category $A$ and a Quillen equivalence

$$
\left(\operatorname{Set}^{\Delta^{\mathrm{op}}}\right)_{S}^{A^{\mathrm{op}}} \underset{R}{\stackrel{L}{\rightleftarrows}} \mathbf{M}
$$

where $\left(\text { Set }^{\Delta^{\mathrm{op}}}\right)_{S}^{A^{\mathrm{op}}}$ is a left Bousfield localization of the projective model structure on the category $\left(\mathbf{S e t}^{\Delta^{\mathrm{op}}}\right){ }_{\text {proj }}^{A^{\text {op }}}$ (here $\mathbf{S e t}^{\Delta^{\mathrm{op}}}$ is taken with the Kan-Quillen model structure). Let $\mathcal{G}=\{L \circ y(a) \mid a \in A\}$ be the image of the representable in $A$ under $L$. For every $X \in \mathbf{M}$ and $a \in A$, the derived mapping space $\mathbb{R} \operatorname{Map}_{\mathbf{M}}(L \circ y(a), X)$ is weakly equivalent to

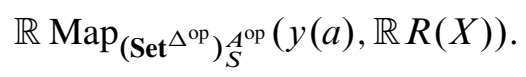

The statement follows from the fact that in $\left(\operatorname{Set}^{\Delta^{\mathrm{op}}}\right)_{S}^{A^{\mathrm{op}}}$, a map $f: X^{\prime} \rightarrow Y^{\prime}$ is a weak equivalence if and only if the induced map

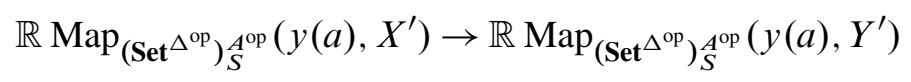

is a weak equivalence for every $a \in A$. 
Assume now that $\mathbf{S}$ still satisfies Assumption 3.2 and is moreover left proper. Let $\mathcal{G}$ be a set of homotopy generators of $\mathbf{S}$ whose existence is given by Lemma 7.2. Without loss of generality, we may assume that the elements of $\mathcal{G}$ are cofibrant. Let $l: I^{\triangleleft} \rightarrow \mathbf{T}$ be an element of $L$. We then consider the composite

$$
\left(I^{\mathrm{op}}\right)^{\triangleright} \stackrel{l^{\mathrm{op}}}{\longrightarrow} \mathbf{T}^{\mathrm{op}} \stackrel{y}{\rightarrow} \mathbf{S e t}^{\mathbf{T}},
$$

where $y$ is the Yoneda embedding. This cocone induces a map

$$
f_{l, G}: \underset{i \in I}{\operatorname{hocolim}} G \otimes y(l(i)) \rightarrow G \otimes y(l(*)),
$$

where $*$ denotes the cone point of $I^{\triangleleft}$, and the homotopy colimit is computed in the model category $\mathbf{S}_{\text {proj }}^{\mathbf{T}}$. Let $H_{L, \mathcal{G}}=\left\{f_{l, G} \mid l \in L, G \in \mathcal{G}\right\}$ be the set of all such maps. We define $\mathbf{h M o d}(\mathbf{T}, L)_{\mathbf{S}}$ to be the left Bousfield localization of $\mathbf{S}_{\text {proj }}^{\mathbf{T}}$ with respect to the maps of $H_{L, \mathcal{G}}$. We have the following characterization of the fibrant objects of $\operatorname{hMod}(\mathbf{T}, L)_{\mathbf{S}}$.

Proposition 7.3 An object $X$ in $\mathbf{S}^{\mathbf{T}}$ is fibrant in $\mathbf{h M o d}(\mathbf{T}, L)_{\mathbf{S}}$ if and only if it is projectively fibrant and for each $l \in L$, the map

$$
X(l(*)) \rightarrow \operatorname{holim}_{i \in I} X(l(i))
$$

is a weak equivalence.

Proof Since the localization only depends on the homotopy type of the maps of $H_{L, \mathcal{G}}$, we may assume, without loss of generality, that the sources of all the maps of $H_{L, \mathcal{G}}$ are cofibrant (note that their targets are already cofibrant). Assume that $X$ is fibrant in $\mathbf{S}_{\text {proj }}^{\mathbf{T}}$. Let $X$. be a simplicial framing of $X$ in $\mathbf{S}_{\text {proj }}^{\mathbf{T}}$. Then for any cofibrant object $A$ of $\mathbf{S}_{\text {proj }}^{\mathbf{T}}$, the simplicial set $\mathbf{S}^{\mathbf{T}}\left(A, X_{\bullet}\right)$ is a model for the derived mapping space $\mathbb{R} \operatorname{Map}_{\mathbf{S}^{\mathbf{T}}}(A, X)$. Moreover, by definition of the projective model structure, for each $t$ in $\mathbf{T}$, the simplicial object $X_{\bullet}(t)$ is a simplicial framing of $X(t)$.

The object $X$ is fibrant in $\operatorname{hMod}(\mathbf{T}, L)_{\mathbf{S}}$ if and only if for any $l \in L$ and $G \in \mathcal{G}$, the map

$$
\mathbf{S}^{\mathbf{T}}\left(G \otimes y(l(*)), X_{\bullet}\right) \rightarrow \mathbf{S}^{\mathbf{T}}\left(\underset{i \in I}{\operatorname{hocolim}} G \otimes y(l(i)), X_{\bullet}\right)
$$

is a weak equivalence. Equivalently, this happens if and only if the map

$$
\mathbf{S}^{\mathbf{T}}\left(G \otimes y(l(*)), X_{\bullet}\right) \rightarrow \underset{i \in I}{\operatorname{holim}} \mathbf{S}^{\mathbf{T}}\left(G \otimes y(l(i)), X_{\bullet}\right)
$$

is a weak equivalence. Equivalently, by definition of the tensor product (see (2-1)), this happens if and only if we have a weak equivalence

$$
\mathbf{S}\left(G, X_{\bullet}(l(*))\right) \rightarrow \underset{i \in I}{\operatorname{holim}} \mathbf{S}^{\mathbf{T}}\left(G, X_{\bullet}(l(i))\right) .
$$


Since this has to be true for any $G$, by Lemma 7.2, we see that $X$ is fibrant if and only if

$$
X(l(*)) \rightarrow \operatorname{holim}_{i \in I} X(l(i))
$$

is a weak equivalence.

Our main theorem takes the following form.

Theorem 7.4 Let $\mathbf{S}$ be a left proper model category satisfying Assumption 3.2. Let $(\mathbf{T}, L)$ be a finite connected limit sketch which is fully faithful in its category of models. Then there is a model structure on $\operatorname{Mod}(\mathbf{T}, L)_{\mathbf{S}}$ in which a map is a fibration (resp. cofibration, weak equivalence) if and only if its image by id $\otimes N$ is a fibration (resp. cofibration, weak equivalence) in $\mathbf{h} \operatorname{Mod}(\mathbf{T}, L)_{\mathbf{S}}$. The functor id $\otimes N$ is then a right Quillen equivalence. Finally, the model structure on $\operatorname{Mod}(\mathbf{T}, L)_{\mathbf{S}}$ is left proper.

Proof This follows immediately from Theorem 5.9.

\subsection{Operads}

We denote by $\mathbf{O p}$ the category of small multicategories in sets. As explained in [21], there exists a category $\Omega$ of trees equipped with a fully faithful functor $\Omega \rightarrow \mathbf{O p}$ inducing a nerve functor $N_{d}: \mathbf{O p} \rightarrow \mathbf{S e t}^{\Omega^{\mathrm{op}}}$. It is straightforward to see that the nerve $N_{d}$ preserves filtered colimits and coproducts. Thus, the pair $(\mathbf{O p}, \Omega)$ satisfies Assumptions 1.1.

We can form the projective model structure on $\mathbf{S}^{\Omega^{\text {op }}}$. Let $\mathcal{G}$ be a set of generators as in Lemma 7.2. Assuming that $\mathbf{S}$ is left proper, we can perform the $\mathbf{S}$-enriched left Bousfield localization of $\mathbf{S}^{\Omega^{\text {op }}}$ with respect to the maps

$$
G \otimes \operatorname{Sc}(T) \rightarrow G \otimes \Omega[T]
$$

for any tree $T$ in $\Omega$ and any $G \in \mathcal{G}$. We denote this model structure by $\mathbf{S}_{\text {Segal }}^{\Omega^{\text {op }}}$. We prove exactly as in Proposition 7.3 that its fibrant objects are the functors $\Omega^{\text {op }} \rightarrow \mathbf{S}$ satisfying the Segal condition. We immediately deduce the following theorem from Theorem 5.9.

Theorem 7.5 The projective and the Segal model structures on $\mathbf{S}^{\Omega^{\text {op }}}$ can each be transferred to a model structure on $\mathbf{S} \otimes \mathbf{O p}$ along the nerve functor id $\otimes N_{d}$. Moreover, in both cases, the functor id $\otimes N_{d}$ is a right Quillen equivalence that preserves and reflects cofibrations. 
Note that if $\mathbf{S}$ is the category $\mathbf{S e t}^{\Delta^{\mathrm{op}}}$ with its usual model structure, the model category $\mathbf{S}_{\text {Segal }}^{\Omega^{\text {op }}}$ is Quillen equivalent to the model structure of dendroidal Segal spaces constructed in [10, Definition 5.4]. In fact, one can show using [14, Proposition 1.7] that the two model structures have the same weak equivalences. In that case, we can construct a further localization $\mathbf{S}_{\text {Rezk }}^{\Omega^{\text {op }}}$ of $\mathbf{S}^{\Omega^{\text {op }}}$ in which the fibrant objects are the fibrant in $\mathbf{S}_{\text {Segal }}^{\Omega^{\text {op }}}$ that are also complete. Theorem 5.9 allows us to transfer this model structure to a Quillen-equivalent model structure on $\mathbf{S} \otimes \mathbf{O p}$, denoted $\mathbf{S} \otimes \mathbf{O} \mathbf{p}_{\text {Rezk }}$. Moreover, the model structure $\mathbf{S}_{\text {Rezk }}^{\Omega^{\text {op }}}$ is itself Quillen equivalent to the model structure constructed in [10, Definition 6.2]. In particular, the $\infty$-category underlying $\mathbf{S} \otimes \mathbf{O} \mathbf{p}_{\text {Rezk }}$ is a model for the $\infty$-category of $\infty$-operads.

\section{3 n-categories}

The category of $n$-categories, denoted $n$ Cat, is defined inductively. The induction is started by defining 1 Cat $=$ Cat. Then, the category $n$ Cat is the category of categories enriched in $(n-1)$ Cat. There is a full subcategory $\Theta_{n}$ (see [22, Corollary 3.10]) of $n$ Cat spanned by compact connected objects. It follows that the pair $\left(n \mathbf{C a t}, \Theta_{n}\right)$ satisfies Assumptions 1.1. Moreover, Rezk inductively constructs a set $\mathcal{T}_{n}^{\mathrm{Se}}$ of maps in Set $_{n}^{\Theta_{n}^{\text {op }}}$ such that the $\Theta_{n}$-spaces that are local with respect to these maps satisfy the suitable form of Segal condition that makes them models for weak $n$-categories. The construction of this set is inductive and rather involved. We refer the reader to Section 5 of [22].

For a model category $\mathbf{S}$ that satisfies Assumption 3.2 and is left proper with set of generators $\mathcal{G}$ (see Lemma 7.2), we can form the left Bousfield localization of $\mathbf{S}_{n}^{\Theta_{n}^{\text {op }}}$ with respect to the set $\bigsqcup_{G \in \mathcal{G}} G \otimes \mathcal{T}_{n}^{\text {Se }}$. We denote this model structure by $\mathbf{S}_{\text {Segal }}^{\Theta_{n}^{\text {op }}}$ and call it the Segal model structure. Applying Proposition 7.3, we immediately see that the fibrant objects in this model structure are the functors $\Theta_{n}^{\text {op }} \rightarrow \mathbf{S}$ satisfying the Segal condition.

Theorem 5.9 immediately yields the following:

Theorem 7.6 The projective and the Segal model structure on $\mathbf{S}_{n}^{\Theta^{\text {op }}}$ can be transferred to a model structure on $\mathbf{S} \otimes n \mathbf{C a t}$ along the nerve functor id $\otimes N$. Moreover, in both cases, the functor id $\otimes N$ is a right Quillen equivalence that preserves and reflects cofibrations.

If $\mathbf{S}$ is the model category of simplicial sets with the Kan-Quillen model structure, then we can construct a further localization of $\mathbf{S}_{n}^{\Theta_{n}^{\text {op }}}$ in which the fibrant objects are fibrant in $\mathbf{S}_{\text {Segal }}^{\Theta_{n}^{\text {op }}}$, and moreover, they are complete. The set of maps with respect to which we need to localize is the set $\mathcal{T}_{n, \infty}$ defined in [22, Section 11.4]. The resulting model structure 
denoted $\mathbf{S}_{\mathrm{Rezk}}^{\Theta_{n}^{\text {op }}}$ is Quillen equivalent to the model structure of $(\infty, n)-\Theta$-spaces defined in [22, Proposition 11.5]. Theorem 5.9 allows us to transfer this model structure to a Quillen-equivalent model structure on $\mathbf{S} \otimes n \mathbf{C a t}$ that we denote by $\mathbf{S} \otimes n \mathbf{C a t}_{\text {Rezk }}$. In particular, the $\infty$-category underlying $\mathbf{S} \otimes n \mathbf{C a t}_{\text {Rezk }}$ is a model for the $\infty$-category of $(\infty, n)$-categories in the sense that it satisfies the axiomatic description of [3].

\section{4 n-fold categories}

The category of $n$-fold categories is the category $\mathbf{C a t}^{\otimes n}$ where Cat denotes the locally presentable category of small categories. The category Cat can be expressed as a reflective subcategory of $\mathbf{S e t}^{\Delta^{\text {op }}}$ via the usual nerve functor Cat $\rightarrow \mathbf{S e t}^{\Delta^{\mathrm{op}}}$. It follows that $\mathbf{C a t}^{\otimes n}$ is a reflective subcategory of $\operatorname{Set}^{\left(\Delta^{n}\right)^{\text {op }}}$; moreover, one easily verifies that the image of $\Delta^{n}$ in $\mathbf{C a t}^{\otimes n}$ consists of compact and connected objects. It follows that the pair $\left(\mathbf{C a t}^{\otimes n}, \Delta^{n}\right)$ satisfies Assumptions 1.1.

We denote by $\Delta\left[k_{1}, k_{2}, \ldots, k_{n}\right]$ the objects of $\operatorname{Set}^{\left(\Delta^{n}\right)^{\text {op }}}$ represented by $\left[k_{1}\right] \times \cdots \times\left[k_{n}\right]$. Note that the object $\Delta\left[k_{1}, \ldots, k_{n}\right]$ is isomorphic to $\Delta\left[k_{1}\right] \otimes \cdots \otimes \Delta\left[k_{n}\right]$ modulo the equivalence of categories $\operatorname{Set}^{\left(\Delta^{n}\right)^{\mathrm{op}}} \simeq\left(\operatorname{Set}^{\Delta^{\mathrm{op}}}\right)^{\otimes n}$.

We denote by $\mathcal{S}$ the set of arrows in $\operatorname{Set}^{\Delta^{\mathrm{op}}}$,

$$
f(n): G(n) \rightarrow \Delta[n],
$$

where $G(n)$ is the object of Set $^{\Delta^{\text {op }}}$ that represents the $n$-fold fiber product $X \mapsto$ $X_{1} \times_{X_{0}} X_{1} \times X_{0} \cdots \times_{X_{0}} X_{1}$ and $f(n)$ represents the Segal map

$$
X_{n} \rightarrow X_{1} \times_{X_{0}} X_{1} \times_{X_{0}} \cdots \times_{X_{0}} X_{1} .
$$

A simplicial set is local with respect to the maps of $\mathcal{S}$ if and only if it is in the essential image of the nerve functor Cat $\rightarrow$ Set $^{\Delta^{\mathrm{op}}}$.

We denote by $\mathcal{S}^{\otimes n}$ the smallest set of arrows of $\operatorname{Set}^{\left(\Delta^{n}\right)^{\text {op }}}$ that contains the arrows

$$
f\left(k_{1}\right) \otimes \mathrm{id}_{\Delta\left[k_{2}\right]} \otimes \cdots \otimes \mathrm{id}_{\Delta\left[k_{n}\right]}
$$

for all $n$-tuples $\left(k_{1}, \ldots, k_{n}\right)$, and that is invariant under the action of $\Sigma_{n}$ on $\operatorname{Set}^{\left(\Delta^{n}\right)^{\text {op }}}$.

As in the previous paragraph, we assume that $\mathbf{S}$ is left proper, and we denote by $\mathcal{G}$ a set of homotopy generators; see Lemma 7.2. We denote by $\mathbf{S}_{\text {Segal }}^{\left(\Delta^{n}\right)^{\text {op }}}$ the left Bousfield localization of the projective model structure on $\mathbf{S}^{\left(\Delta^{n}\right)^{\text {op }}}$ with respect to the maps in $\bigsqcup_{G \in \mathcal{G}} G \otimes \mathcal{S}^{\otimes n}$. Using Proposition 7.3, we see that an object is fibrant in $\mathbf{S}_{\text {Segal }}^{\left(\Delta^{n} \text { op }\right.}$ if it is projectively fibrant and if the simplicial object of $\mathbf{S}$ obtained by fixing all the variables but one is fibrant in $\mathbf{S}_{\text {Segal }}^{\Delta^{\mathrm{op}}}$. Theorem 5.9 then implies the following theorem. 
Theorem 7.7 The projective and the Segal model structure on $\mathbf{S}^{\left(\Delta^{n}\right)^{\text {op }}}$ can be transferred to a model structure on $\mathbf{S} \otimes \mathbf{C a t}^{\otimes n}$ along the nerve functor id $\otimes N$. Moreover, in both cases, the functor id $\otimes N$ is a right Quillen equivalence that preserves and reflects cofibrations.

Example 7.8 The authors of [9] construct a $\Delta^{n}$-space encoding the cobordism $n$-fold $\infty$-category. Using our main Theorem 7.7 , we can rigidify this model to an equivalent strict $n$-fold category in the category of simplicial sets with the same homotopy type. An explicit construction of a strict $n$-fold category of cobordisms is also done in [8].

\subsection{Monads with (connected compact) arities}

Given a monad $T$ on a category $\mathbf{B}$, we denote its category of algebras by $\operatorname{Alg}(T)$. It is known that if $\mathbf{B}$ is finitely locally presentable and $T$ is finitary, the category $\operatorname{Alg}(T)$ is also finitely locally presentable and thus presented by a finite limit sketch. However, in applications, one would like to have an explicit description of a sketch describing the theory considered.

When $\mathbf{B}$ is a presheaf category, the theory of monads with arities introduced by Berger, Melliès and Weber (see [24; 5]) provides a way to recover a fully faithful sketch describing $\operatorname{Alg}(T)$ from the data of $T$ and a sufficiently nice dense subcategory $\Theta_{0}$ of $\mathbf{B}$. The aim of this section is to recall this theory and to show how our theorem applies in this situation.

We fix a small category $C$ and a monad $T$ over Set $^{C^{\text {op }}}$. Given a dense full subcategory $\Theta_{0}$ of Set $C^{\text {op }}$ and an object $A \in$ Set $^{C^{\text {op }}}$, the $\Theta_{0}$-cocone over $A$ is just the canonical cocone $\left(\Theta_{0} \downarrow A\right)^{\triangleright} \rightarrow$ Set $^{C^{\text {op }}}$ with tip $A$. The monad $T$ is with arities $\Theta_{0}$ if the composite $N_{\Theta_{0}} T$ sends $\Theta_{0}$-cocones in Set ${ }^{\text {op }}$ to colimits-cocones in $\mathbf{S e t}^{\Theta_{0}^{\text {op }}}$; see [5, Definition 1.8].

Let $\Theta_{0}$ be a dense full subcategory of Set $^{C^{\text {op }}}$ that provides arities for $T$, and denote the associated nerve functor by $N_{\Theta_{0}}:$ Set $^{C^{\text {op }}} \rightarrow$ Set $^{\Theta_{0}^{o p}}$. If we denote by $\Theta_{T}$ the full image of $\Theta_{0}$ in $\operatorname{Alg}(T)$, with nerve functor $N_{\Theta_{T}}$, we have the following diagram:

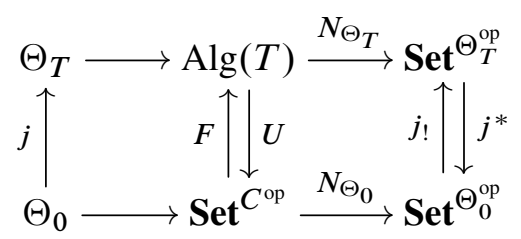

Berger, Melliès and Weber showed that if $T$ is with arities $\Theta_{0}$, then $\Theta_{T}$ is dense in $\operatorname{Alg}(T)$, and the essential image of $N_{\Theta_{T}}$ is spanned by the presheaves $F$ such that $j^{*}(F)$ is in the essential image of $N_{\Theta_{0}}$; see [5, Theorem 1.10] and [24, Theorem 4.10]. 
Since we are interested in finite connected sketches, we restrict our attention to a more specific situation:

Assumptions 7.9 Let $C, \Theta_{0}$ and $T$ be as above; we make the following assumptions:

(1) $T$ is a monad with arities $\Theta_{0}$.

(2) $\Theta_{0}$ contains $C$.

(3) For every $a \in \Theta_{0}$, the comma category $C \downarrow a$ is finite and connected. This implies in particular that all the objects of $\Theta_{0}$ are compact and connected in $\mathbf{S e t}^{C^{\text {op }}}$.

For every $a \in \Theta_{0}$, let $l_{a}:(C \downarrow a)^{\triangleright} \rightarrow \Theta_{0}$ be the canonical $C$-cocone over $a$, and let

$$
L=\left\{l_{a}^{\mathrm{op}}:\left(a \downarrow C^{\mathrm{op}}\right)^{\triangleleft} \rightarrow \Theta_{0}^{\mathrm{op}} \mid a \in \Theta_{0}\right\}
$$

be the set of all the opposite cones.

The maps in $\mathbf{S e t}^{\Theta_{0}^{\text {op }}}$ of the kind

$$
a \rightarrow \operatorname{colim}_{c \in C \downarrow a} h_{c}
$$

for some $a \in \Theta_{0}$ are called the Segal maps.

The essential image of $N_{\Theta_{0}}$ is spanned by those $F \in$ Set $^{\Theta_{0}^{\text {op }}}$ such that the canonical map induced by $l_{a}$,

$$
F(a) \cong \operatorname{Set}^{\Theta_{0}^{\mathrm{op}}}(a, F) \rightarrow \operatorname{Set}^{\Theta_{0}^{\mathrm{op}}}\left(\operatorname{colim}_{c \in C \downarrow a} c, F\right) \cong \lim _{c \in C \downarrow a} F(c),
$$

is an isomorphism for every $a \in \Theta_{0}$ (see [24, Proposition 4.13]); in other words, Set $C^{\text {op }}$ is equivalent to the category of models of the finite connected limit sketch $\left(\Theta_{0}^{\mathrm{op}}, L\right)$.

Therefore, diagram (7-2) above is equivalent to the following diagram:

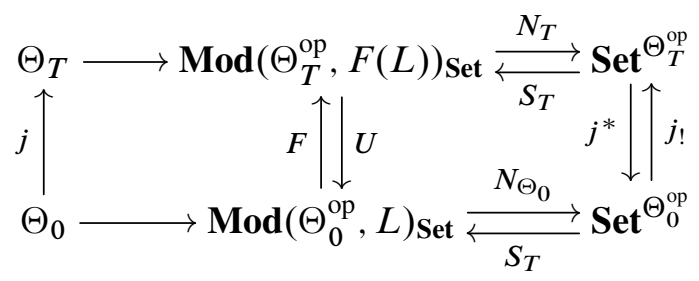

In particular, the category $\operatorname{Alg}(T)$ can be described as the category of models for the fully faithful finite connected limit sketch $(\Theta, j(L))$. 
If we let $\mathbf{S}$ be as in Section 7.1, tensoring with $\mathbf{S}$, we get the following diagram of Quillen adjunctions:

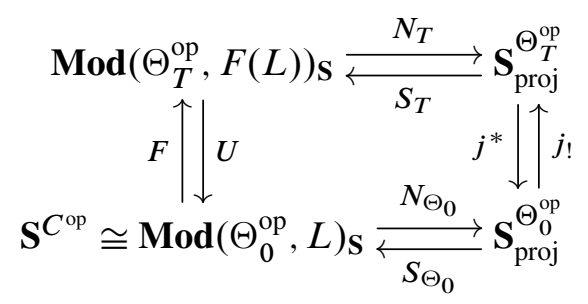

where the horizontal adjunctions are Quillen equivalences. Localizing on both rows at the Segal maps as in Section 7.1, the diagram remains a diagram of Quillen adjunctions:

$$
\begin{aligned}
& \left(\operatorname{Mod}\left(\Theta_{T}^{\mathrm{op}}, j(L)\right)_{\mathbf{S}}\right)_{\text {Segal }} \underset{S_{T}}{\stackrel{N_{T}}{\rightleftarrows}} \mathbf{h M o d}\left(\Theta_{T}^{\mathrm{op}}, j(L)\right)_{\mathbf{S}}
\end{aligned}
$$

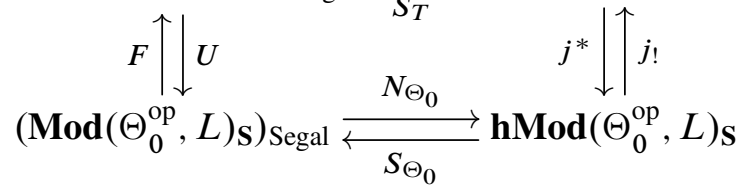

where the rows are Quillen equivalences.

All the examples presented in the previous sections can all be regarded as instances of the monad with arities, as explained in [5]. For example, Cat is the category of algebras for a monad $T$ over the category of graphs Set $^{20 p}$, where 2 is the category with set of objects $\{0,1\}$ and with only two arrows different from the identities, both from 0 to 1 .

Let $\Theta_{0}$ be the full subcategory of Set $^{20 p}$ spanned by the finite linear graphs, ie those $G \in$ Set $^{20 \mathrm{pp}}$ such that $G(0)$ and $G(1)$ are finite, $|G(0)|=|G(1)|+1$, and $G(s)$ and $G(t)$ are injective with different images. $\Theta_{0}$ satisfies Assumptions 7.9 and is equivalent to $\Delta_{\text {out }}$, the wide subcategory of $\Delta$ spanned by all the maps $f$ between finite ordinals such that $f(i-1)=f(i)-1$ for every $i>0$ in the domain of $f$. The full image of $\Theta_{0}$ in Cat is equivalent to $\Delta$, and the Segal maps correspond to the classical Segal maps.

Similarly, the dendroidal category $\Omega$ can be obtained from the monad over the category of symmetric multigraphs which has $\mathbf{O p}$ as the category of algebras and has the category of trees (and tree embeddings) as arities; see [24] and [18]. The Segal maps correspond exactly to the maps of type (7-1).

The category of $n$-categories of Section 7.3 is the category of algebras for a monad $T$ over ( $n$-truncated) globular sets; the category of $n$-globular pasting diagrams provides arities for $T$, it satisfies Assumptions 7.9, and its full image in $n$ Cat is isomorphic to $\Theta_{n}$. 
The category $\omega$ Cat of strict infinity categories is also the category of algebras for a monad $T$ over (nontruncated) globular sets. The category of globular pasting diagrams satisfies Assumptions 7.9 (with respect to $T$ ). We refer the reader to [5, Sections 2.11 and 3.12], [4] and [20, Section 8.1] for details.

Other examples of monads with arities are given in [5] and [24]. One example of a monad with arities that satisfies Assumptions 7.9 is the monad for properads over the presheaf category of digraphical species presented in [19], which has the category of connected acyclic graphs and étale maps (presented in [loc. cit.]) as arities.

\section{References}

[1] J Adámek, J Rosický, Locally presentable and accessible categories, London Math. Soc. Lect. Note Ser. 189, Cambridge University Press (1994) MR

[2] C Barwick, On left and right model categories and left and right Bousfield localizations, Homology, Homotopy Appl. 12 (2010) 245-320 MR

[3] C Barwick, C Schommer-Pries, On the unicity of the homotopy theory of higher categories, preprint (2011) arXiv

[4] C Berger, Iterated wreath product of the simplex category and iterated loop spaces, Adv. Math. 213 (2007) 230-270 MR

[5] C Berger, P-A Melliès, M Weber, Monads with arities and their associated theories, J. Pure Appl. Algebra 216 (2012) 2029-2048 MR

[6] J E Bergner, Three models for the homotopy theory of homotopy theories, Topology 46 (2007) 397-436 MR

[7] J E Bergner, C Rezk, Comparison of models for $(\infty, n)$-categories, I, Geom. Topol. 17 (2013) 2163-2202 MR

[8] M Bökstedt, I Madsen, The cobordism category and Waldhausen's K-theory, preprint (2011) arXiv

[9] D Calaque, C Scheimbauer, A note on the $(\infty, n)$-category of cobordisms, preprint (2015) arXiv

[10] D-C Cisinski, I Moerdijk, Dendroidal Segal spaces and $\infty$-operads, J. Topol. 6 (2013) 675-704 MR

[11] D Dugger, Combinatorial model categories have presentations, Adv. Math. 164 (2001) 177-201 MR

[12] B Guillou, J P May, Enriched model categories and presheaf categories, preprint (2011) arXiv

[13] P S Hirschhorn, Model categories and their localizations, Mathematical Surveys and Monographs 99, Amer. Math. Soc., Providence, RI (2003) MR 
[14] G Horel, A model structure on internal categories in simplicial sets, Theory Appl. Categ. 30 (2015) 704-750 MR

[15] J F Jardine, Local homotopy theory, Springer, New York (2015) MR

[16] P T Johnstone, Topos theory, London Math. Soc. Monogr. 10, Academic Press, London (1977) MR

[17] G M Kelly, Basic concepts of enriched category theory, London Math. Soc. Lect. Note Ser. 64, Cambridge University Press (1982) MR

[18] J Kock, Polynomial functors and trees, Int. Math. Res. Not. 2011 (2011) 609-673 MR

[19] J Kock, Graphs, hypergraphs, and properads, Collect. Math. 67 (2016) 155-190 MR

[20] T Leinster, Higher operads, higher categories, London Math. Soc. Lect. Note Ser. 298, Cambridge University Press (2004) MR

[21] I Moerdijk, I Weiss, Dendroidal sets, Algebr. Geom. Topol. 7 (2007) 1441-1470 MR

[22] C Rezk, A Cartesian presentation of weak n-categories, Geom. Topol. 14 (2010) 521-571 MR

[23] C Simpson, Homotopy theory of higher categories, New Mathematical Monographs 19, Cambridge University Press (2012) MR

[24] M Weber, Familial 2-functors and parametric right adjoints, Theory Appl. Categ. 18 (2007) 665-732 MR

Institute for Mathematics, Astrophysics, and Particle Physics, Radboud University Nijmegen, Heyendaalseweg 135, 6525 AJ Nijmegen, Netherlands

Max Planck Institute for Mathematics

Vivatsgasse 7, D-53111 Bonn, Germany

g.caviglia@math.ru.nl, geoffroy.horel@gmail.com

http://www.math.ru.nl/ gcaviglia/, http://geoffroy.horel.org/

Received: 13 November $2015 \quad$ Revised: 16 May 2016 